\title{
有机多孔聚咔唑的制备及性能研究进展
}

\author{
操强陈琦韩宝航* \\ (国家纳米科学中心 北京 100190)
}

\begin{abstract}
摘要 聚咔唑具有刚性主链和共轭富电子体系, 既有利于形成永久性多孔材料, 又可增强被吸附物与吸附剂之间的相 互作用，还具有特异的光电性能. 因此，近年来有机多孔聚咔唑材料的研究成为有机多孔材料领域中的一个热点. 有 机多孔聚咔唑一般具有较大的比表面积和稳定的孔结构, 其制备方法简单多样, 多孔性可调控, 而且可以保持良好的 光学电学性质, 在气体存储与分离、有机蒸气吸附、催化、传感及有机电子学等方面具有潜在的应用价值. 就有机多 孔聚咔唑材料的制备而言, 常用的制备方法是以氧化偶联反应和 Friedel-Crafts 反应为代表的合成方法, 还有一些如氰 基三聚和碳一碳偶联反应等其他的合成方法. 本文主要介绍近几年有机多孔聚咔唑的制备方法和性能研究与应用方面 的最新进展.
\end{abstract}

关键词 咔唑; 有机多孔聚合物; 制备; 性能

\section{Recent Advance in Organic Porous Polycarbazoles: Preparation and Properties}

\author{
Cao, Qiang Chen, Qi Han, Baohang* \\ (National Center for Nanoscience and Technology, Beijing 100190)
}

\begin{abstract}
Polycarbazole has rigid backbone and conjugated electron rich system, which are beneficial to form permanent porous materials, enhance interactions between adsorbate and adsorbent, and exhibit intrinsic optical and electrical performance. As a novel kind of porous materials, organic porous polycarbazoles possess high specific surface area and permanent porosity, which have drawn great interests owing to the advantages in synthetic diversity, pore size controllability, optical and electrical properties. The preparation of organic porous polycarbazoles has recently been developed rapidly because of their great potential applications in gas storage, separation, vapor adsorption, catalysis, sensing and organic electronics. As for preparative methods of the organic porous polycarbazoles, carbazole-based oxidative coupling polymerization and Friedel-Crafts alkylation are the representative methods. Some other synthetic methods such as nitrile-based trimerization of aromatic nitriles and classic carbon-carbon coupling polymerization. Recently, a facile method for the preparation of hypercrosslinked organic porous polycarbazoles via $\mathrm{FeCl}_{3}$-promoted one-step oxidative coupling reaction and Friedel-Crafts alkylation in one pot has also been reported. According to the summarized results of porosity and adsorption performance, micro/mesoporous conjugated polycarbazole with high porosity can be obtained via molecular structure tuning. The Brunauer-Emmett-Teller specific surface area of porous polycarbazole is up to $2440 \mathrm{~m}^{2} \cdot \mathrm{g}^{-1}$. The adsorption performance of some organic porous polycarbazoles not only can be comparable with that of the known porous organic polymers with ultrahigh specific surface area, such as PAF-1 and PNN-4, but also can be competitive with the best reported results for porous organic polymers, activated carbons, and metal-organic frameworks under the same conditions. Herein, recent advance such as synthetic methods, properties, and applications in organic porous polycarbazoles has been reviewed.

Keywords carbazole; porous organic polymer; preparation; property and application
\end{abstract}

\section{1 引言}

目前为大家所熟知的多孔材料有沸石、多孔碳和二 氧化硅等无机类材料 ${ }^{[1 \sim 3]}$ 以及金属-有机框架化合物 (metal-organic frameworks, MOFs). 有机多孔聚合物 (porous organic polymers, POPs) 是一类近期发展起来的 高度交联的无定形、具有纳米孔隙的聚合物. 它们具有 较高的比表面积和孔容. 因此, 有机多孔材料在气体吸
附 ${ }^{[4]}$ 、分离以及非均相催化 ${ }^{[5]}$ 方面有比较广泛的应用. 根 据其结构特点的不同, 有机多孔聚合物可分为共价有机 骨架 ${ }^{[6 \sim 10]}$ 、自具微孔聚合物 ${ }^{[11 ~ 13]}$ 、共轭微孔聚合物 ${ }^{[14,15]}$ 、 超交联聚合物 ${ }^{[16]}$ 、多孔芳香族框架 ${ }^{[17 \sim 19]}$ 和多孔聚合物 网络 ${ }^{[20]}$ 等. 这些多孔材料通常由轻质元素组成, 主要是 碳、氢、氧、氮、硼等. 根据不同合成方法, 分类介绍 有机多孔材料研究进展的综述在国内已有报道 ${ }^{[21,22]}$, 汇 总了有机多孔聚合物的经典制备方法以及所采用的有

* E-mail: hanbh@nanoctr.cn; Tel.: 010-82545576; Fax: 010-82545576

Received February 11, 2015; published April 13, 2015.

Project supported by the National Natural Science Foundation of China (Nos. 21274033 and 21374024) and the National Science and Technology Major Project of the Ministry of Science and Technology of China (No. 2014CB932200).

项目受国家自然科学基金(Nos. 21274033, 21374024)和科技部国家重大科研项目(No. 2014CB932200)资助. 
机分子建筑块, 并简单总结了其在气体存储、分离和非 均相催化方面的应用. 全球二氧化碳的排放量在过去几 十年里急剧上升, 被广泛认为是全球气候变暖的主要原 因之一. 通过碳捕获和储存(Carbon Capture and Sequestration, $\mathrm{CCS})^{[23]}$ 的方法并进一步利用二氧化碳成为降低 大气二氧化碳含量的重要举措之一. 有机多孔材料在二 氧化碳吸附和储存方面的研究备受关注.

咔唑作为一种很重要的含氮杂环化合物, 它对热、 酸和碱都比较稳定, 而且具有刚性主链和共轭富电子体 系, 既有利于形成永久性多孔材料, 又可增强被吸附物 与吸附剂之间的相互作用, 其含氮结构可大大提高对二 氧化碳酸性气体的吸附能力. 近几年含咔唑有机多孔材 料备受关注, 常用的制备方法是氧化偶联聚合反应和 Friedel-Crafts 反应, 还有一些其他的合成方法如氧基三 聚和碳一碳偶联反应等. 所制备的有机多孔聚咔唑一般 具有较大的比表面积和稳定的孔结构, 多孔性可调控, 因此在气体存储与分离、有机蒸汽吸附、催化方面具有 潜在的应用价值. 此外, 有机多孔聚咔唑可以保持良好 的光学电学性质, 具有特异的光电性能, 在化学生物传 感和有机电子学方面的应用也有新的突破. 本文在重点 介绍有机多孔聚咔唑制备方法的基础上, 总结了材料的 小分子吸附与分离、催化以及光电方面的性能与应用.

\section{2 有机多孔聚咔唑的制备}

目前, 有机多孔材料的制备研究主要集中在材料的 结构设计与合成方法探索. 一方面, 材料的结构具有一 定的可设计性, 构筑有机多孔材料的反应单体具有结构 和种类的丰富性, 不同的构筑单元影响材料的结构及性 质; 另一方面, 制备有机多孔材料的聚合反应类型具有<smiles>c1ccc2c(c1)[nH]c1ccccc12</smiles>

M-1
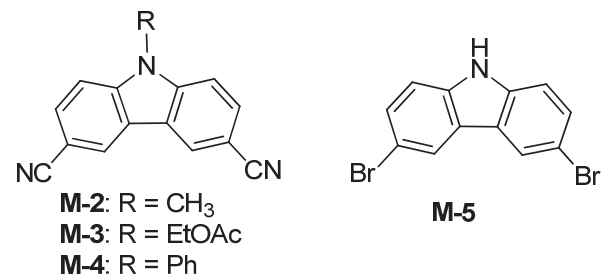

M-5

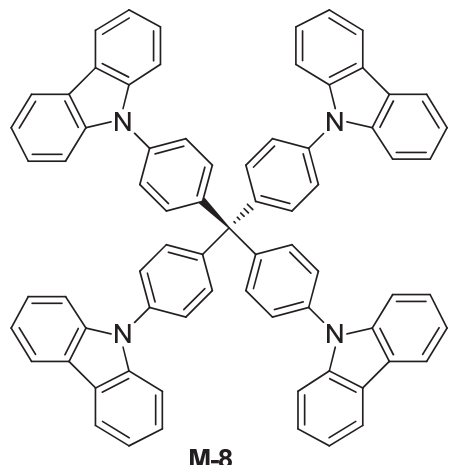

多样性，一种新的聚合反应类型的应用不仅可以拓展有 机多孔材料的制备方法，丰富材料结构的多样性，而且 可以更好的推动材料相关性能的开发与研究. 就有机多 孔聚咔唑材料的制备而言，常用的制备方法是以氧化偶 联反应和 Friedel-Crafts 反应为代表的合成方法(表 1), 还有一些如氰基三聚和碳一碳偶联反应等其他的合成方 法. 根据制备方法的不同, 研究人员设计合成了多种含 咔唑基团的单体，结构如图 1 所示，它们一般是具有非 平面结构的刚性有机分子. 希望通过调节有机单体分子 的大小、长度和结构类型等方法, 调控所制备多孔聚咔 唑材料的孔道结构和比表面积、孔容与孔径分布等孔隙 率参数. 对于氧化偶联反应的制备方法而言，单体中咔 唑取代基的个数对所制备多孔材料的比表面积有重要 影响. 一般含三个以上咔唑取代基的单体 $(\mathbf{C z}-7 \sim \mathbf{C z}-9$ 和 M-10)可制备出高比表面积(1500 2500 $\left.\mathrm{m}^{2} \cdot \mathrm{g}^{-1}\right)$ 的材 料, 相应材料的孔容也比较高 $\left(1.35 \sim 2.04 \mathrm{~cm}^{3} \cdot \mathrm{g}^{-1}\right)$, 这 是由于多咔唑取代的单体具有较多的反应位点，更有利 于形成无限网络结构. 具有类似结构的单体如 $\mathbf{C z - 8} \sim$ Cz-10, 可通过调节联接臂的长度来调节所制备材料的 孔径. CPOP-8 和 CPOP-10 都是微孔材料, 而 CPOP-9 是介孔材料, 微孔率不足 $5 \%$, 它具有最大的比表面积 达到了 $2400 \mathrm{~m}^{2} \cdot \mathrm{g}^{-1}$ 以上, 孔径分布主要在 $2.5 \sim 5.0 \mathrm{~nm}$. 对于 Friedel-Crafts 反应的制备方法而言, 有的反应需要 外加交联剂，有的反应单体本身就含有交联基团，所制 备材料的孔隙率参数没有明显的规律，比表面积一般在 $700 \sim 1200 \mathrm{~m}^{2} \cdot \mathrm{g}^{-1}$, 个别材料的比表面积高达 $2250 \mathrm{~m}^{2} \bullet$ $\mathrm{g}^{-1}$. 利用氰基三聚和碳一碳偶联反应制备的多孔材料的 比表面积不是很高, 不过氰基三聚反应中可通过改变单 体所含取代基的结构来调节材料的孔径分布及孔容.
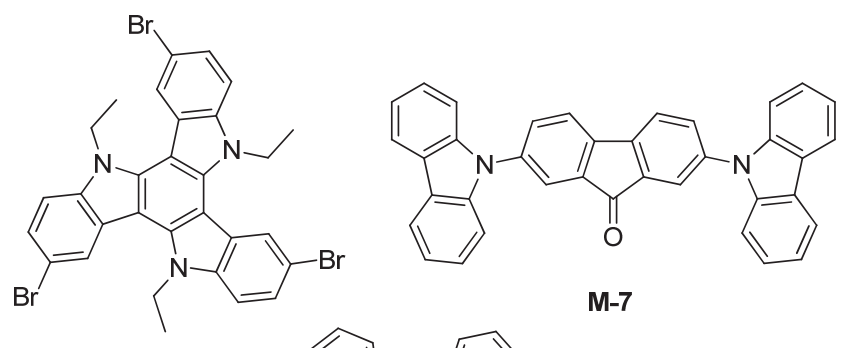

M-7

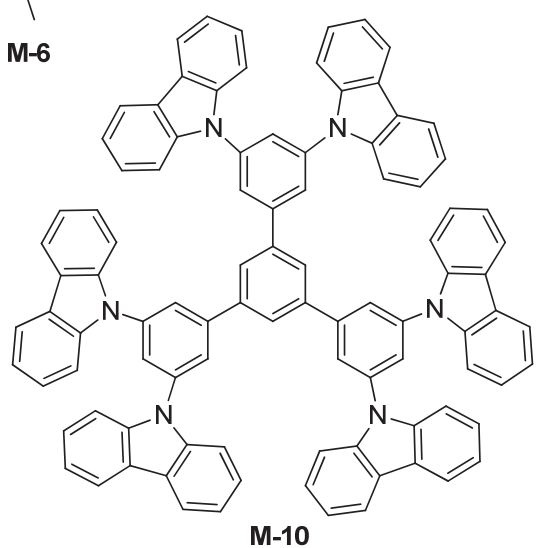



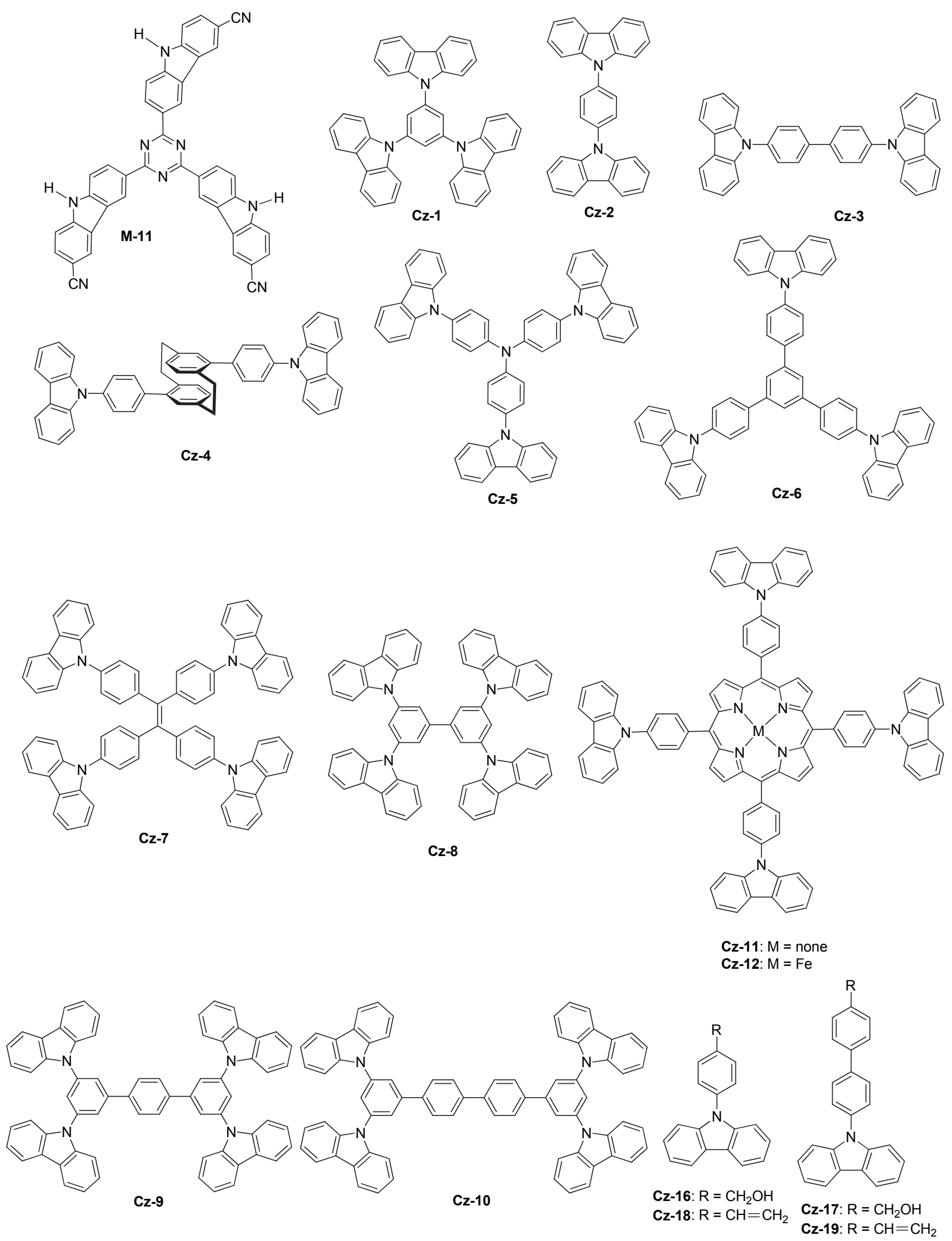

图 1 用于制备有机多孔聚咔唑的部分单体

Figure 1 Part of monomers for preparation of the organic porous polycarbazoles 
表 1 制备多孔聚咔唑的不同单体及方法对比表面积、孔径和孔容的影响

Table 1 The effects of monomers and preparative methods on specific surface area, pore size and pore volume

\begin{tabular}{|c|c|c|c|c|c|c|}
\hline 单体 & 聚合物 & 制备方法 & $S_{\mathrm{BET}} /\left(\mathrm{m}^{2} \cdot \mathrm{g}^{-1}\right)$ & $\mathrm{PSD}^{a} / \mathrm{nm}$ & $V_{\text {tot }}^{b} /\left(\mathrm{cm}^{3} \cdot \mathrm{g}^{-1}\right)$ & Ref. \\
\hline $\mathrm{Cz}-1$ & CPOP-1 & 氧化偶联反应 & 2220 & 0.62 & 1.293 & [26] \\
\hline $\mathrm{Cz}-2$ & CPOP-2 & 氧化偶联反应 & 510 & 0.59 & 0.352 & [27] \\
\hline $\mathrm{Cz}-3$ & CРОР-3 & 氧化偶联反应 & 630 & 0.60 & 0.530 & [27] \\
\hline $\mathrm{Cz}-4$ & CРОР-4 & 氧化偶联反应 & 660 & 0.63 & 0.361 & {$[27]$} \\
\hline $\mathrm{Cz}-5$ & CРOP-5 & 氧化偶联反应 & 1050 & 0.60 & 0.940 & [27] \\
\hline $\mathrm{Cz}-6$ & СРОР-6 & 氧化偶联反应 & 980 & 0.63 & 0.772 & [27] \\
\hline $\mathrm{Cz}-8$ & CPOP-8 & 氧化偶联反应 & 1610 & 0.63 & 1.71 & {$[28]$} \\
\hline $\mathrm{Cz}-9$ & CPOP-9 & 氧化偶联反应 & 2440 & $0.63,2.5 \sim 5.0$ & 2.04 & {$[28]$} \\
\hline $\mathrm{Cz}-10$ & CPOP-10 & 氧化偶联反应 & 1110 & 0.63 & 0.76 & [28] \\
\hline Cz-11 & CPOP-11 & 氧化偶联反应 & 1320 & $0.61 \sim 1.33$ & 1.13 & [29] \\
\hline $\mathrm{Cz}-12$ & CPOP-12 & 氧化偶联反应 & 1180 & $0.59 \sim 1.31$ & 1.05 & [29] \\
\hline M-9 & MFCMP-1 & 氧化偶联反应 & 843 & $0.69 \sim 1.84$ & 0.52 & [31] \\
\hline M-8 & TPTCz & 电化学氧化偶联反应 & - & 0.90 & - & [33] \\
\hline M-1 & TSP-1 & Friedel-Crafts 反应 & 562 & 0.6 & 0.33 & [39] \\
\hline M-7 & TFMSA & Friedel-Crafts 反应 & 2250 & - & 2.16 & [36] \\
\hline M-9 & TSP-2 & Friedel-Crafts 反应 & 913 & 2.2 & 0.82 & [39] \\
\hline $\mathrm{Cz}-2$ & CPOP-13 & 氧化偶联和 Friedel-Crafts 反应 & 890 & $0.63,0.66$ & 0.468 & {$[37]$} \\
\hline $\mathbf{C z - 3}$ & CPOP-14 & 氧化偶联和 Friedel-Crafts 反应 & 820 & $0.63,0.66$ & 0.416 & {$[37]$} \\
\hline $\mathrm{Cz}-1$ & CPOP-15 & 氧化偶联和 Friedel-Crafts 反应 & 1190 & 0.63 & 1.575 & [37] \\
\hline $\mathrm{Cz}-16$ & CPOP-16 & 氧化偶联和 Friedel-Crafts 反应 & 780 & 0.59 & 0.59 & {$[38]$} \\
\hline $\mathrm{Cz}-17$ & CPOP-17 & 氧化偶联和 Friedel-Crafts 反应 & 700 & 0.59 & 0.48 & {$[38]$} \\
\hline $\mathrm{Cz}-18$ & CPOP-18 & 氧化偶联和 Friedel-Crafts 反应 & 1040 & 0.52 & 0.77 & {$[38]$} \\
\hline Cz-19 & CPOP-19 & 氧化偶联和 Friedel-Crafts 反应 & 1130 & 0.59 & 0.80 & [38] \\
\hline M-11 & NPTF-1 & 氧基三聚反应 & 1187 & - & 0.93 & {$[41]$} \\
\hline M-2 & NOP-19 & 氰基三聚反应 & 982 & $0.8 \sim 1.8$ & 0.58 & {$[41]$} \\
\hline M-5 & CMP-22 & Sonogashira-Hagihara 偶联反应 & 779 & - & 1.40 & {$[24]$} \\
\hline M-6 & ТСВ-СМР & Yamamoto 偶联反应 & 1280 & $0.8 \sim 1.5$ & 0.923 & [43] \\
\hline
\end{tabular}

${ }^{a}$ Data calculated from nitrogen adsorption-desorption isotherms with the NLDFT method; ${ }^{b}$ Total pore volume determined from the $\mathrm{N}_{2}$ isotherm at $P / P_{0}=0.99$.

\section{1 通过氧化偶联反应制备有机多孔聚咔唑}

有机多孔共轭聚合物一般是通过以 SonogashiraHagihara 反应 ${ }^{[24]}$ 和 Suzuki 反应 ${ }^{[25]}$ 为代表的碳-碳偶联聚 合反应通过形成芳基-芳基之间的共价键来制备的. 然 而, 这些方法通常需要先合成含多卤代芳环、多炔基或 多嗍酸功能化的有机单体分子, 合成纯化步骤繁琐, 所 使用的贵金属催化剂成本相对较高; 更重要的是这些反 应官能团在最终制备的材料中有残留, 对材料结构和性 能的影响难以确定. 考虑到咔唑的苯环类似联苯、二苯 胺的苯环结构, 有较高的电子云密度, 在无水三氯化铁 的作用下, 咔唑基团之间在 3,6-位置可以发生高效的氧 化偶联反应, 无需其它官能团的参与. 2012 年, 本课题 组 ${ }^{[26]}$ 首次通过由三氯化铁催化的基于咔唑氧化偶联聚 合反应制备得到 Brunauer-Emmett-Teller (BET)比表面 积高达 $2220 \mathrm{~m}^{2} \cdot \mathrm{g}^{-1}$ 的微孔聚咔唑 CPOP-1(图式 1). 这种 制备方法同时具有以下优点: (1)反应温度为室温; (2)催 化剂廉价易得; (3)只需要一种单体参与聚合; (4)产率高 于 $90 \%$; (5)单体和聚合物不含卤素、硼酸、炔基等其它 官能团. 相对于目前有机多孔材料的其它制备方法而
言, 此方法不仅是一种制备思路上的拓展, 更为有机多 孔材料大规模的生产奠定了基础.

我们还合成了一系列的含咔唑的单体结构, 利用具 有非平面刚性结构的 $2 \mathrm{D}$ 或 $3 \mathrm{D}$ 单体合成了一系列共轭 多孔咔唑聚合物 CPOP-2 CPOP-12(图式 2). 通过调节 有机单体分子的大小、长度和结构类型等, 可有效地调 控所制备有机多孔材料的孔道结构和比表面积、孔容与 孔径分布等孔隙率参数. 其中, CPOP-2 C CPOP-7 ${ }^{[27]}$ 多 孔材料的 BET 比表面积随着相应单体中咔唑取代基的 个数增加而增高, 介于 $510 \sim 1430 \mathrm{~m}^{2} \cdot \mathrm{g}^{-1}$ 之间. 这表明 单体中可参与聚合的反应位点越多, 越有利于提高聚合 的效果，从而有助于制备出高比表面积的多孔聚合物. 对于同结构类型的单体而言, 调节联接臂的长度, 也可 有效调控材料的孔隙率参数. CPOP-8 和 CPOP-10 都是 微孔材料，它们的 BET 比表面积介于 $1100 \sim 1700$ $\mathrm{m}^{2} \cdot \mathrm{g}^{-1}$ 之间. 而 CPOP-9 ${ }^{[28]}$ 是介孔材料，微孔率不足 $5 \%$, 它具有最大的比表面积，达到了 $2400 \mathrm{~m}^{2} \cdot \mathrm{g}^{-1}$ 以上，孔径 分布主要在 $2.5 \sim 5.0 \mathrm{~nm}$ 之间. 通过基于咔唑的氧化偶 合聚合也比较容易合成具有特殊功能化的多孔聚合物, 

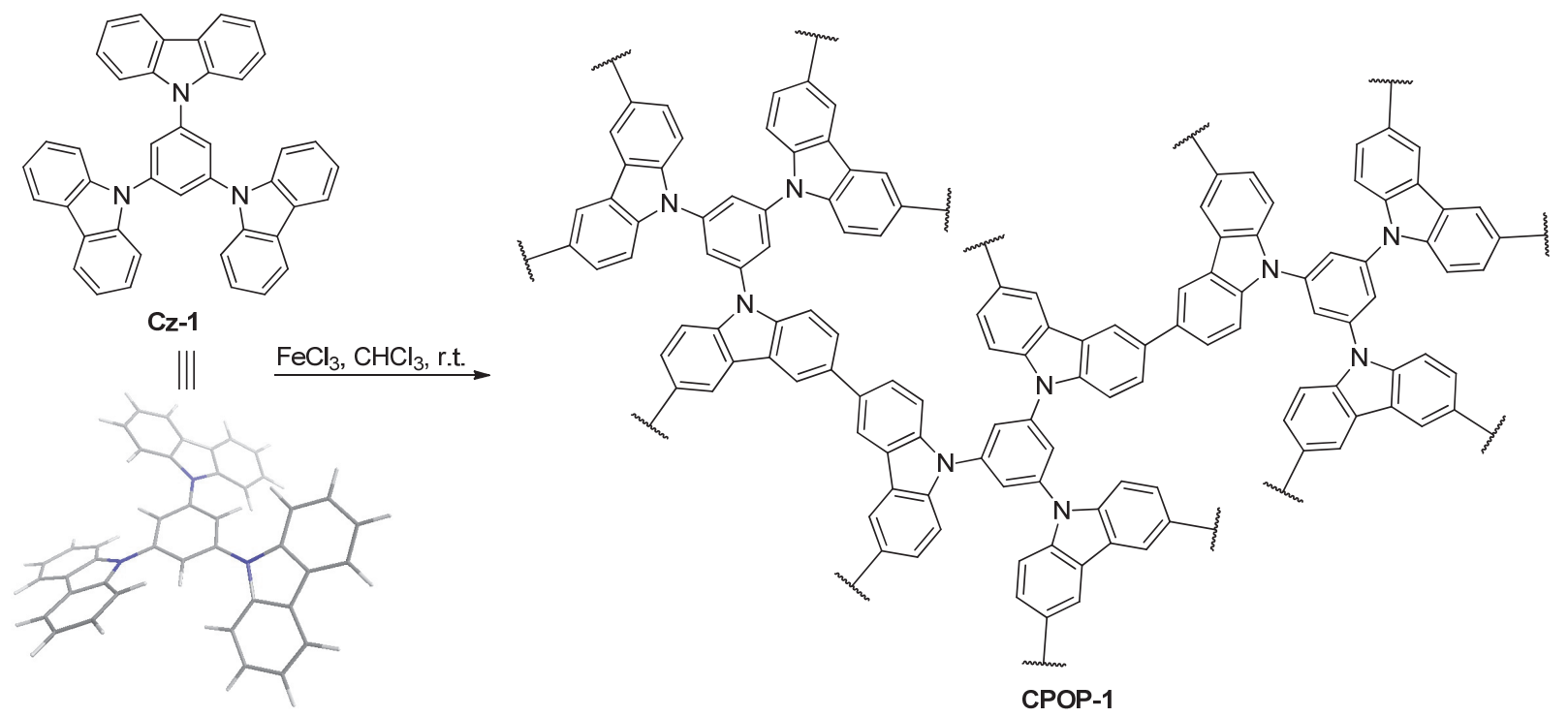

图式 1 共轭多孔聚咔唑 CPOP-1 的合成

Scheme 1 Preparation of conjugated porous polycarbazole CPOP-1

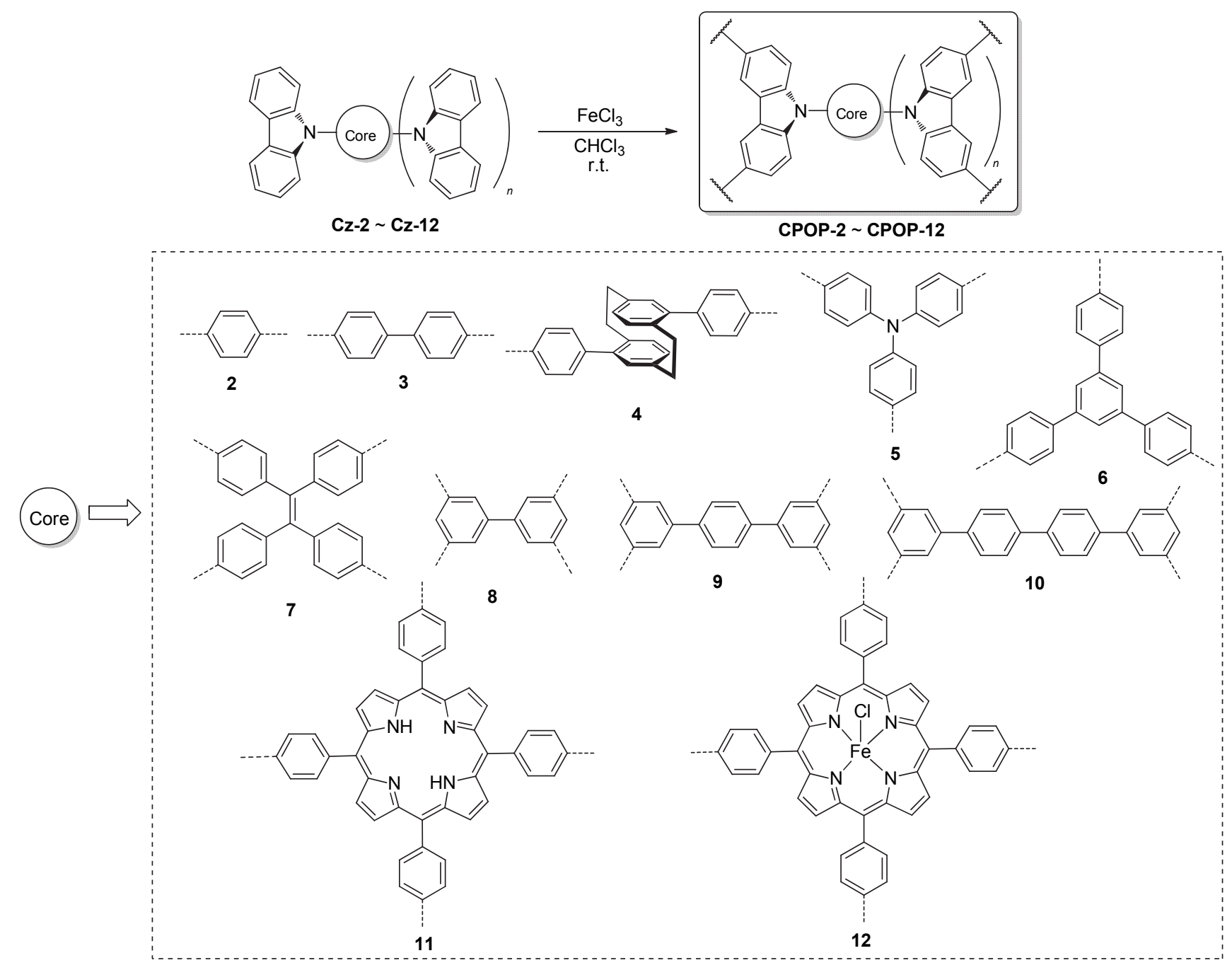

图式 2 共轭多孔聚咔唑 CPOP-2 CPOP-12 的制备

Scheme 2 Preparation of conjugated porous polycarbazoles CPOP-2 $\sim$ CPOP-12 
例如含卟啉多孔共轭聚合物 CPOP-11 和 CPOP-12, 其 中多孔聚卟啉 CPOP-12 的 BET 比表面积高达 1180 $\mathrm{m}^{2} \cdot \mathrm{g}^{-1}$. 因为其含铁卟啉结构, 可以应用于非均相催化 ${ }^{[29]}$.

2014 年, Yang 课题组 ${ }^{[30]}$ 利用三氯化铁氧化偶联聚 合制备了 P-1 和 P-2 两种新型的共轭微孔模型网络(图式 3). 单体 M-7(芴酮)被噻吩的格氏试剂修饰后形成芴叔 醇单体, 可以将化学位点由四个变成五个, 而且将极性 基团 $\mathrm{OH}$ 引入到构建模块中. P-2 的 BET 比表面积(1220 $\left.\mathrm{m}^{2} \cdot \mathrm{g}^{-1}\right)$ 是 $\mathbf{P}-\mathbf{1}\left(610 \mathrm{~m}^{2} \cdot \mathrm{g}^{-1}\right)$ 的两倍, 并且孔容也是 $\mathbf{P}-\mathbf{1}$ $\left(0.95, P / P_{0}=0.99\right)$ 的 1.63 倍. 但这两个聚合物的孔径分 布基本上相近 $(1.19 \mathrm{~nm})$, 这说明了孔径可能由刚性咔 唑一咔唑骨架的尺寸决定的, 不是由聚合程度来决定的.

Liu 课题组 ${ }^{[31]}$ 报道了利用三维结构的刚性有机单体 2,4,6-三咔唑-1,3,5-三嗪自身的高效氧化偶联聚合反应, 形成了结构稳定并含有无限网络孔道的咔唑类有机多 孔聚合物 MFCMP-1(图式 4). 该材料是一种新型的三<smiles>Cc1ccc2c(c1)c1ccccc1n2-c1ccc2c(c1)C(=O)c1cc(-n3c4ccc(C)cc4c4cc(C)ccc43)ccc1-2</smiles>
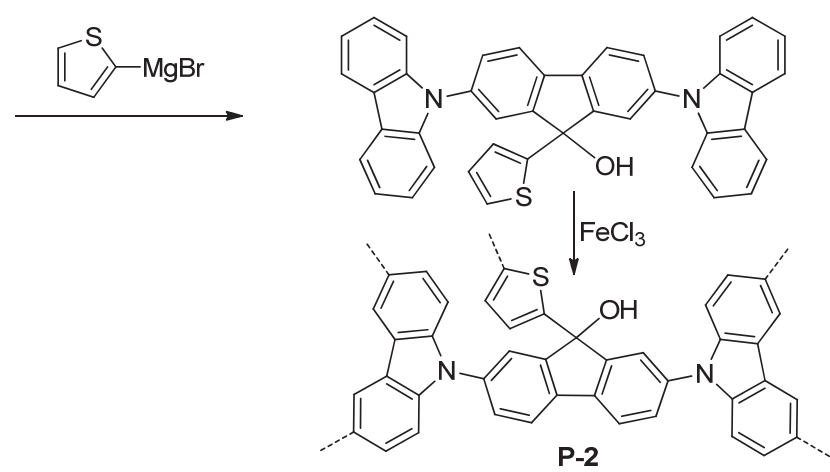

图式 3 P-1 和 P-2 网络结构的合成

Scheme 3 Preparation of P-1 and P-2 polymer networks

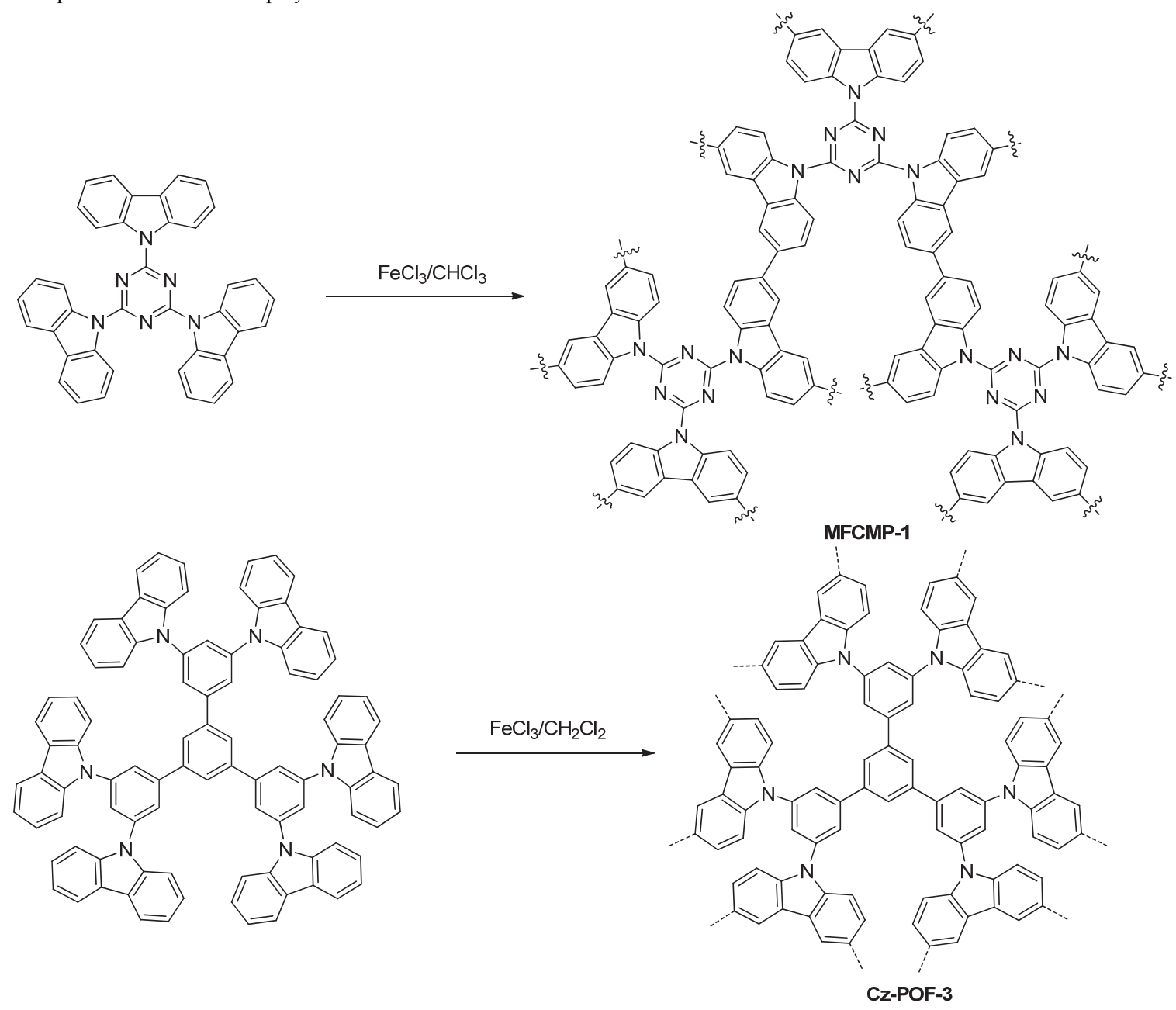

图式 4 微孔聚咔唑 MFCMP-1 和 Cz-POFs 的制备

Scheme 4 Preparation of microporous polycarbazoles MFCMP-1 and Cz-POF-3 
维 $\pi$-共轭聚合骨架, 它能够形成稳定的微孔结构, 并且 具有高发光性能以及丰富的氮活化位点. 近期, Zhang 等 ${ }^{[32]}$ 报道了通过三氯化铁氧化偶联制备咔唑类多孔骨 架聚合物 Cz-POFs. 研究发现提高构建单体中咔唑取代 基的数量的可以显著提高 Cz-POFs 的孔隙率. 其中 Cz-POF-3(图式 4)的比表面积接近 $2000 \mathrm{~m}^{2} \cdot \mathrm{g}^{-1}$.

除了化学氧化偶联聚合的方法制备多孔聚咔唑外, 电化学聚合也被应用于制备共轭多孔聚咔唑. 2013 年, $\mathrm{Li}$ 和 $\mathrm{Ma}$ 等 ${ }^{[33]}$ 报道了利用电化学聚合制备共轭微孔聚咔 唑薄膜, 他们以四咔唑取代的四苯甲烷为聚合前体通过 电化学聚合制备出了相应的多孔聚咔唑薄膜 TPTCz-CMP(图式 5), 并进一步构筑成有机电子器件应 用于 PLEDs 中. 后来, Jiang 等 ${ }^{[34]}$ 也利用电化学方法以三 咔唑取代的三苯基苯为聚合前体可控制备了共轭微孔 聚咔唑 TPBCz-CMP 薄膜(图式 5). 值得一提的是该薄 膜的厚度可以控制, 而且可以用作衬底或独立的薄膜使 用，已应用于化疗和生物传感.

\section{2 通过 Friedel-Crafts 反应制备超交联有机多孔聚咔}

唑

Friedel-Crafts 反应常用于制备超交联有机多孔聚合 物，一般在 Lewis 酸的催化下，通过卤代烃、醇、烯、 醛或酮与芳香环之间发生的亲电取代反应制备超交联
聚合物，该方法的优势在于合成路线的高性价比，这对 于大规模生产尤为重要。2013 年, Wasielewski 和 Kanatzidis 课题组 ${ }^{[35]}$ 报道用对苯二甲醛与咔唑直接制备 有机多孔聚合物 Car-POF(图式 6). 该材料在聚合初期 存在稳定的未成对的电子, 之后证实在这类材料中存在 自由基，这说明了该材料在光学半导体的潜在应用. 该 研究工作基于一个简单的合成路线制备有机多孔材料, 突出了对苯二甲醛作为单体用于合成价格低廉官能化 多孔聚合物的重要意义. 同年, Scherf课题组 ${ }^{[36]}$ 利用 2,7双( $N$-咔唑基)-9-芴酮在酸性条件下自缩合制备微孔聚 合网络结构 MPNs(图式 6), 比表面积高达 $2250 \mathrm{~m}^{2} \cdot \mathrm{g}^{-1}$.

鉴于三氯化铁催化氧化偶联反应和三氯化铁催化 Friedel-Crafts 烷基化反应的优越性，同时考虑到咔唑及 其衍生物的特殊结构和电化学性质, 我们课题组 ${ }^{[37]}$ 利 用三种结构相对简单的咔唑类单体，以二甲氧基甲烷为 交联剂, 在三氯化铁的催化下同时发生氧化偶联反应和 Friedel-Crafts 烷基化反应，一锅法制备出了超交联的多 孔聚咔唑类有机多孔材料 CPOP-13 CPOP-15(图式 7). 同一种催化剂三氯化铁起到两种催化作用，一是作为咔 唑苯环之间的氧化偶联聚合反应的催化剂, 三氯化铁中 三价铁的氧化性起主导作用; 二是咔唑类单体与交联剂 之间的 Friedel-Crafts 烷基化反应中，三氯化铁作为路易
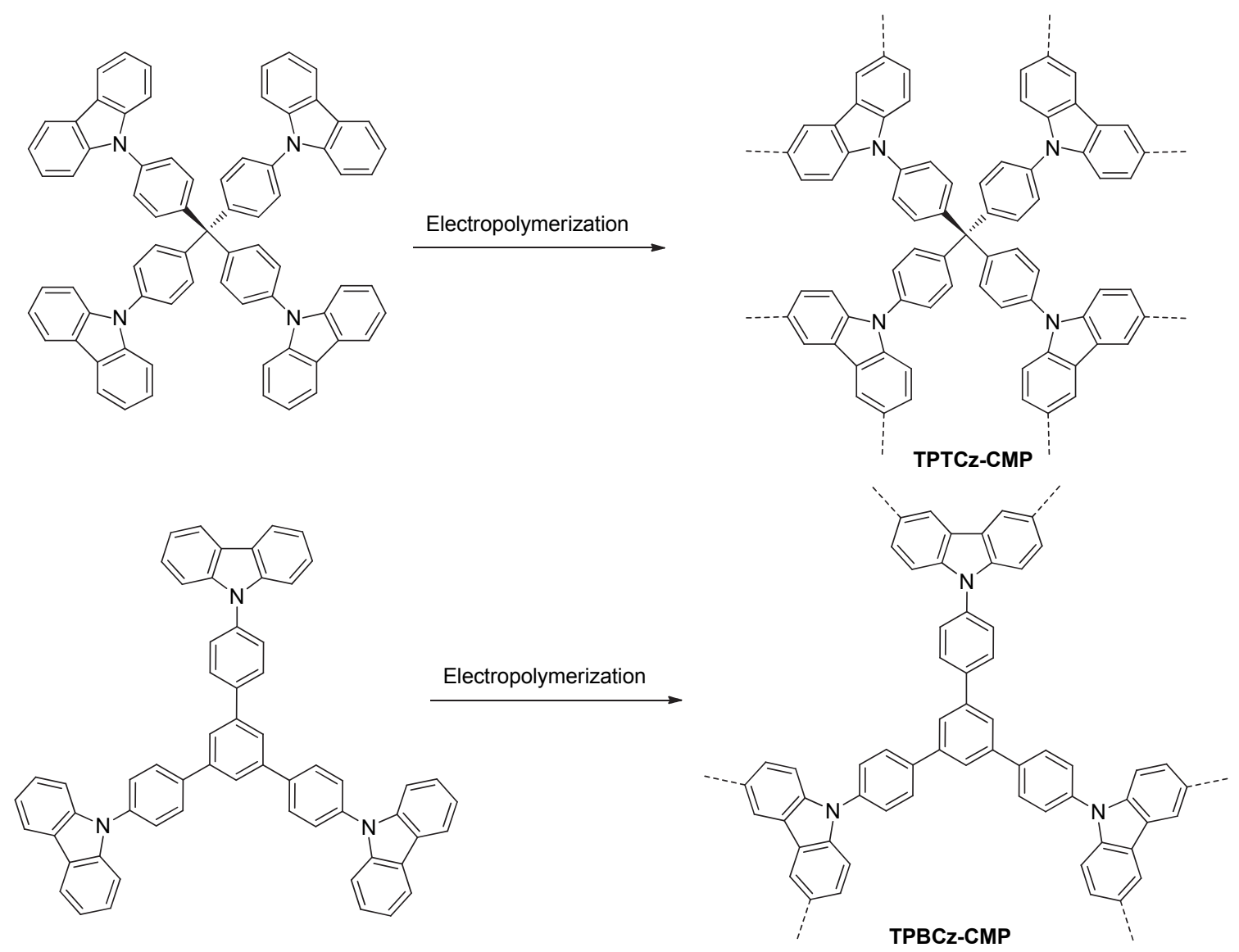

图式 5 电化学聚合制备 TPTCz-CMP 和 TPBCz-CMP 薄膜

Scheme 5 Preparation of TPTCz-CMP and TPBCz-CMP by electropolymerization 


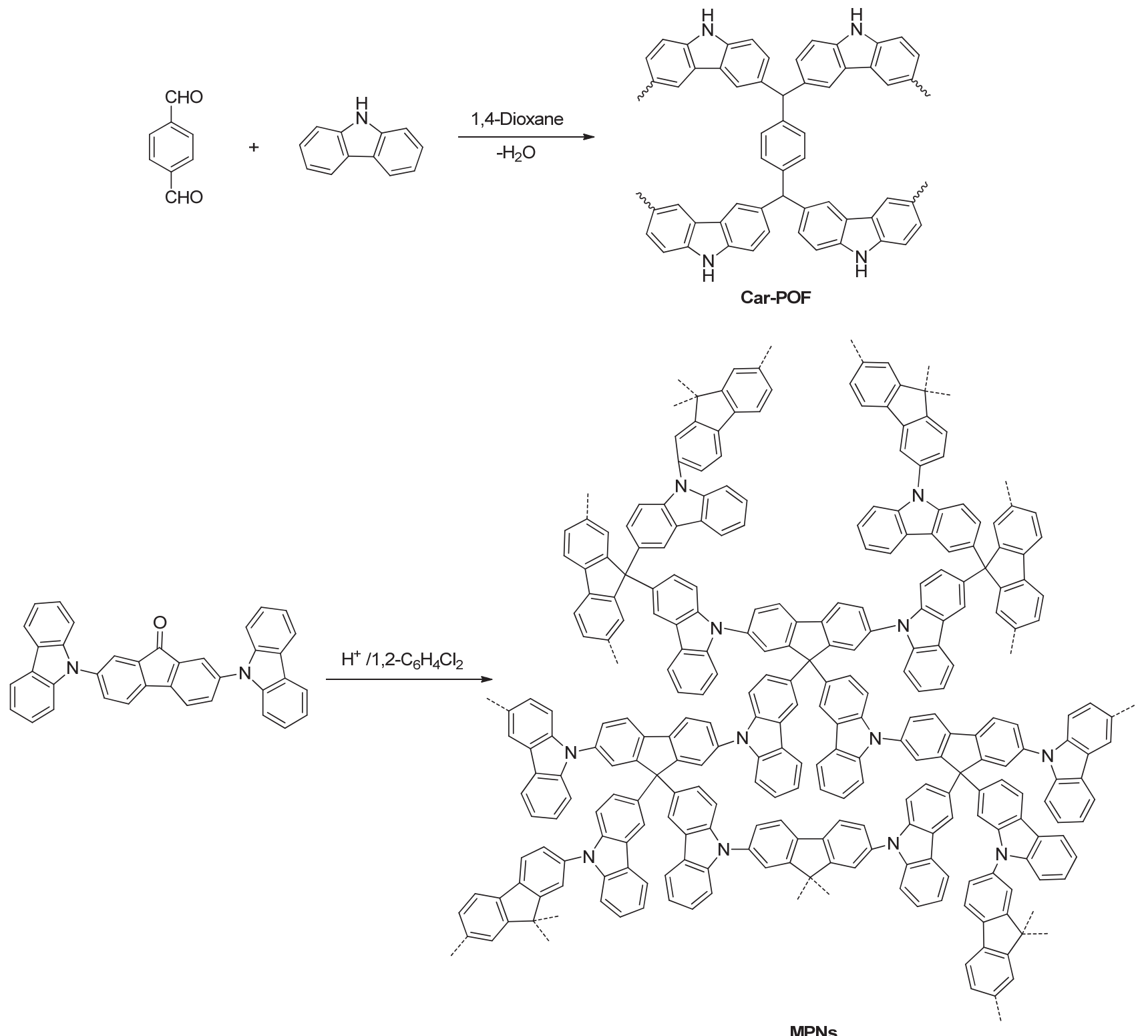

图式 6 利用 Friedel-Crafts 反应制备 Car-POFs 和 MPNs

Scheme 6 Preparation of Car-POFs and MPNs by Friedel-Crafts reaction

斯酸催化 Friedel-Crafts 烷基化反应的发生. 由于两个反 应同时可以发生, 使在聚合物的制备过程中, 单体的活 性反应位点得到更为充分的利用, 进而使形成的聚合物 网络结构更为规整, 有利于提高其各方面性质. 近期, 我们又利用羟甲基或乙烯基取代的咔唑单体为原料, 无 需外加交联剂, 在三氯化铁的催化下, 通过氧化偶联反 应和 Friedel-Crafts 反应一步法制备了基于咔唑的超交 联多孔有机聚合物 CPOP-16 CPOP-19(图式 8$)^{[38]}$. 目 前, 超交联聚合物已被用于气体和有机蒸汽的吸附, 并 且可以用于从水中除去有机化合物.

Liu 课题组 ${ }^{\left[{ }^{[3]}\right]}$ 报道通过简单的 Friedel-Crafts 反应制 备一种多孔的三嗪和咔唑聚合物 TSPs(图式 9). 所得到 的多孔骨架对二氧化碳表现出较好的吸附性. Kim 等 ${ }^{[40]}$ 也同样以二甲氧基甲烷为交联剂通过 Friedel-Crafts 反
应合成微孔有机聚合物. 他们对多孔材料的吸附性能以 及选择性做了进一步的研究, 比表面积高达 1020 $\mathrm{m}^{2} \cdot \mathrm{g}^{-1}$, 孔径分布比较均匀在 $0.6 \sim 0.8 \mathrm{~nm}$ 之间, 并且有 较好的物理化学稳定性.

\section{3 通过其它方法制备有机多孔聚咔唑}

\subsection{1 氭基三聚}

2014 年, Pan 课题组 ${ }^{[41]}$ 以双氰基取代的咔唑为单体 利用氰基三聚成三嗪的反应制备了一系列多孔聚咔唑， 并实现了通过引入三种不同的附加官能团(甲基、乙酸 乙酯基和苯基)来控制孔隙率(图式 10). 所合成的有机 多孔聚咔唑(NOPs)有良好的热稳定性和高的比表面积. 含苯基框架聚合物 NOP-21 对二氧化碳的吸附量最高达 $12.3 \mathrm{wt} \%$ (在 $273 \mathrm{~K}, 1.0 \mathrm{bar}$ ). 此外, 基于理想吸附溶液 
理论，含乙酸乙酯框架聚合物 NOP-20 对二氧化碳/氮气 体系表现出较好的选择性. 通过控制孔隙率构建高性能<smiles>c1ccc2c(c1)[nH]c1ccccc12</smiles>

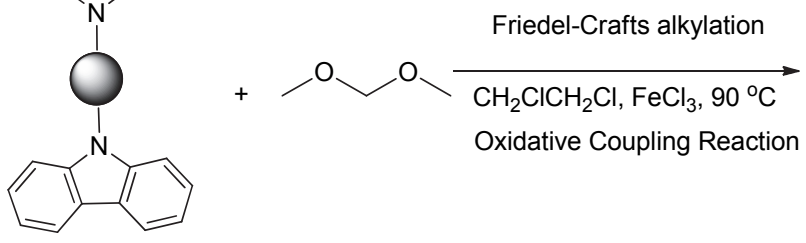

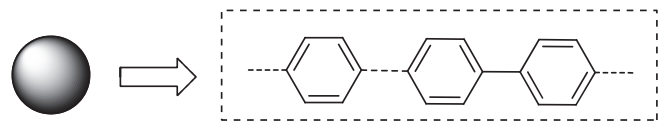

的有机多孔二氧化碳吸附剂，这些结果表明了其良好的 可行性.

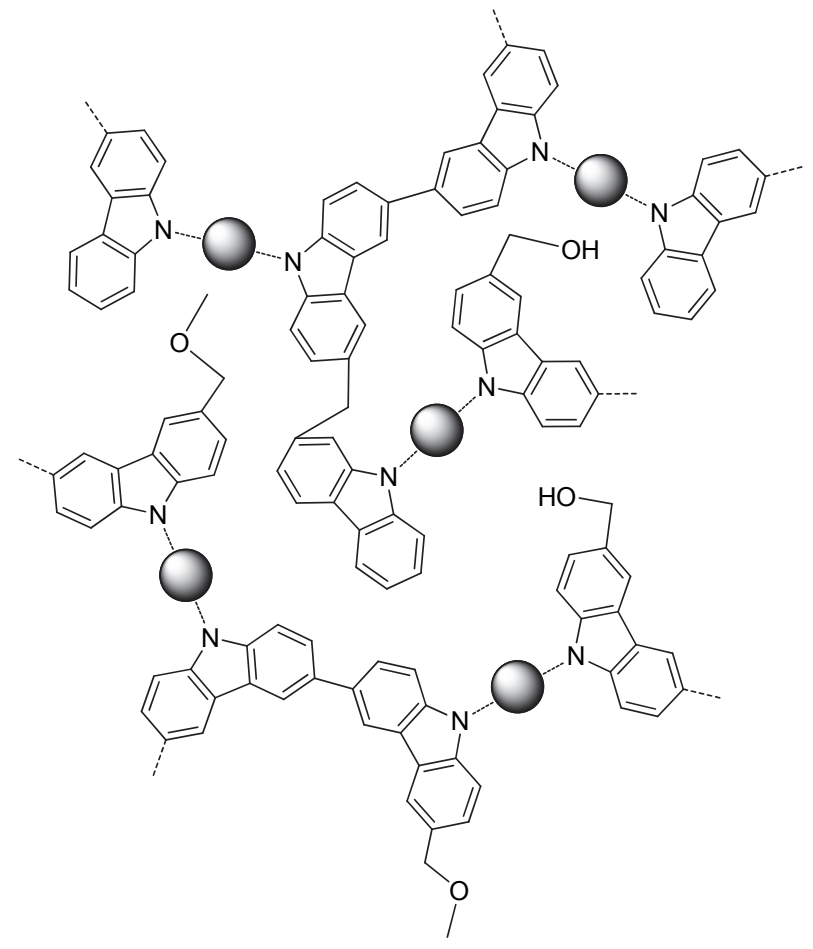

CPOP-13, CPOP-14

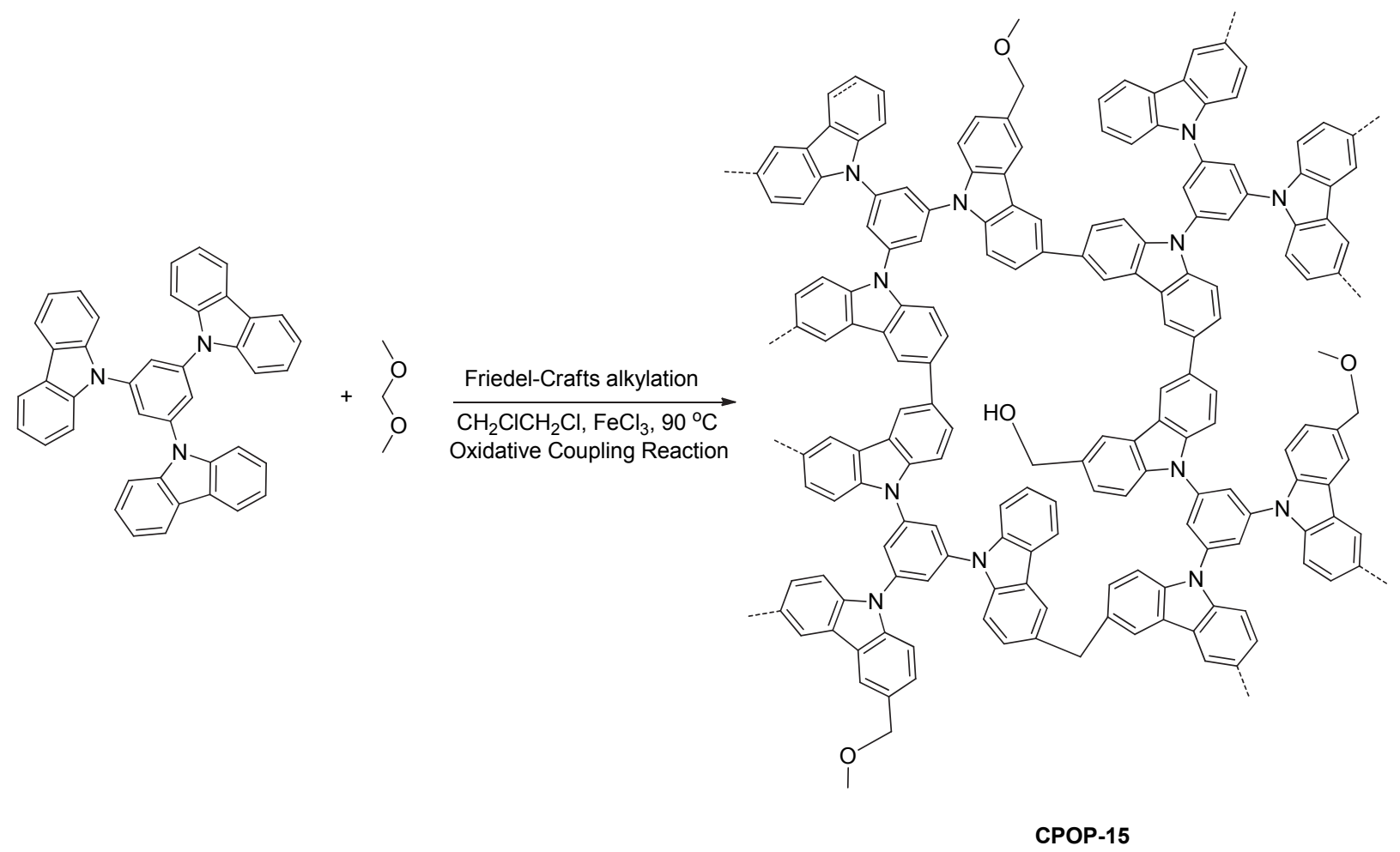

图式 7 超交联多孔聚咔唑 CPOP-13 CPOP-15 的合成

Scheme 7 Preparation of hypercrosslinked porous polycarbazoles CPOP-13 $\sim$ CPOP-15 

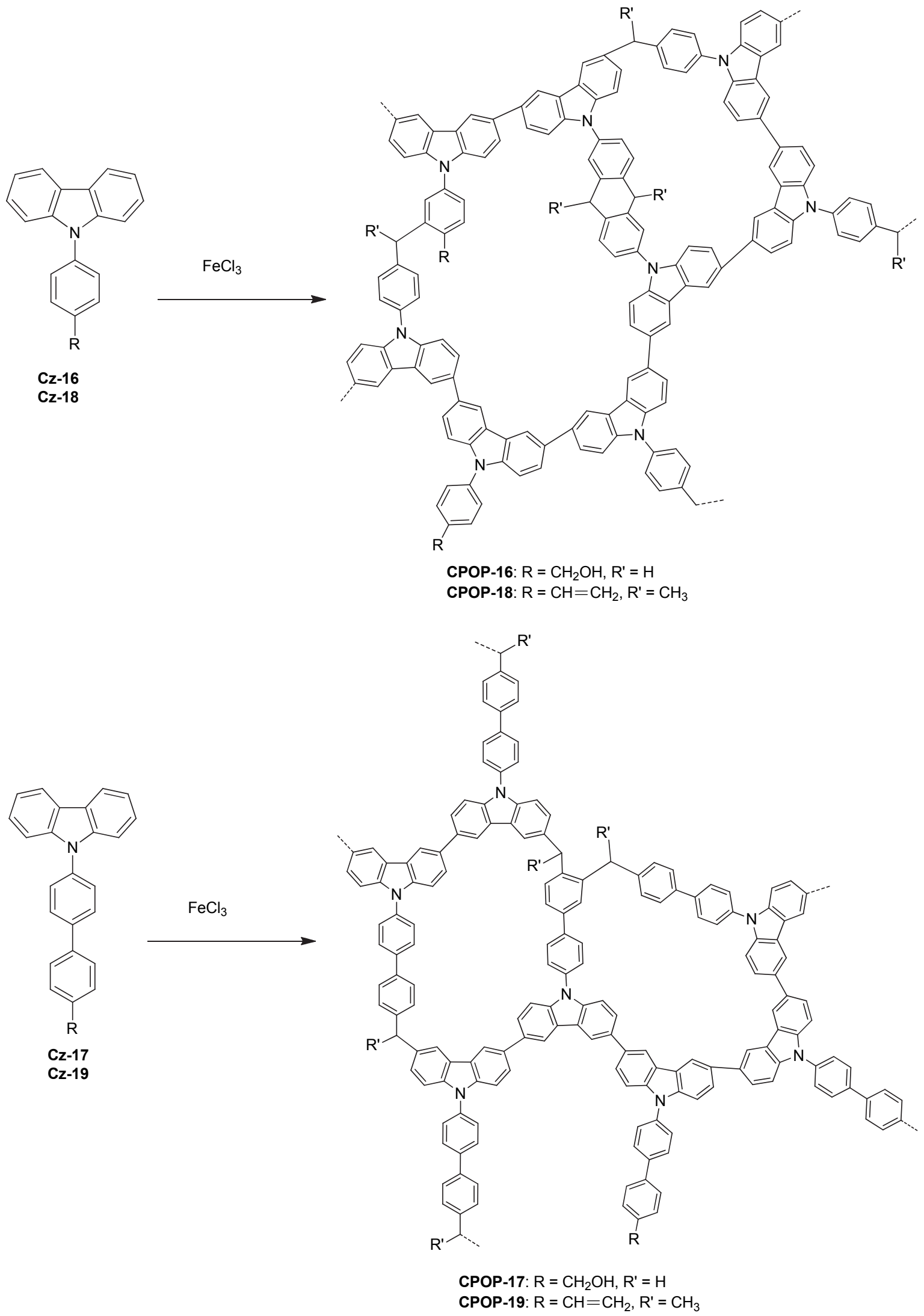

图式 8 超交联多孔聚咔唑 CPOP-16 CPOP-19 的合成

Scheme 8 Preparation of hypercrosslinked porous polycarbazoles CPOP-16 $\sim$ CPOP-19 
<smiles>c1ccc2c(c1)[nH]c1ccccc12</smiles><smiles>COCOC</smiles>

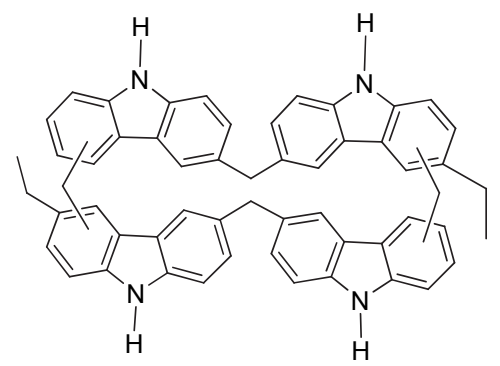<smiles>c1ccc2c(c1)c1ccccc1n2-c1nc(-n2c3ccccc3c3ccccc32)nc(-n2c3ccccc3c3ccccc32)n1</smiles><smiles>COCCOCOC</smiles><smiles>CCc1ccc2c(c1)c1cc(CC)ccc1n2-c1nc(-n2c3ccc(CC)cc3c3cc(CC)ccc32)nc(-n2c3ccc(CC)cc3c3cc(CC)ccc32)n1</smiles>

图式 9 超交联多孔聚咔唑 TSPs 的合成

Scheme 9 Preparation of hypercrosslinked porous polycarbazoles TSPs<smiles>[R]n1c2ccc(C#N)cc2c2cc(C#N)ccc21</smiles><smiles>[CH2-]</smiles>

图式 10 制备有机多孔聚咔唑 NOPs

Scheme 10 Preparation of polymers NOPs

此外，他们还报道利用热不稳定的氧聚合构建高性 能稳定孔隙材料 NPTNs (图式 11), 将咔唑、二苯并呋喃 和二苯并噻吩等杂环引入到三嗪类网络中探究杂环对 气体吸附性能的影响 ${ }^{[42]}$. 用 3,6-二氰基咔唑、3,6-二氧基 苯并呋喃和 3,6-二氰基苯并噻吩为原料在温和的条件下 合成热稳定性较好的中间体, 然后在高温条件下合成三 嗪类聚合物. 它们具有很高物理化学稳定性和比较理想 的 BET 比表面积.

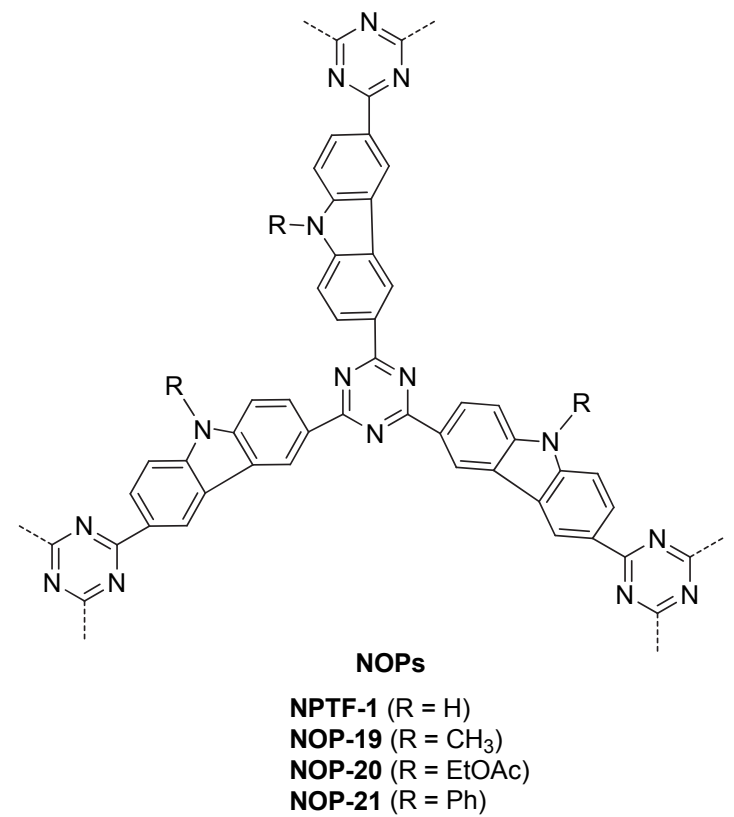

\subsubsection{Sonogashira-Hagihara 偶联反应}

2009 年, Cooper 等 ${ }^{[24]}$ 采用 Sonogashira-Hagihara 偶 联反应合成共轭微孔聚合物(Eq. 1)，以 1,3,5-三苯乙炔 和 3,6-二溴咔唑为单体制备多孔材料 CMP-22, 比表面 积高达 $780 \mathrm{~m}^{2} \cdot \mathrm{g}^{-1}$. 由于多孔材料的表面化学性质是随 着单体的结构而变化, 因此可以通过改变单体来调控材 料的物理性质, 例如疏水性. 另外, 通过进一步改变该 网络结构的疏水性, 实现对染料吸附性能的调控, 使得 该材料可应用于催化和分离等领域. 


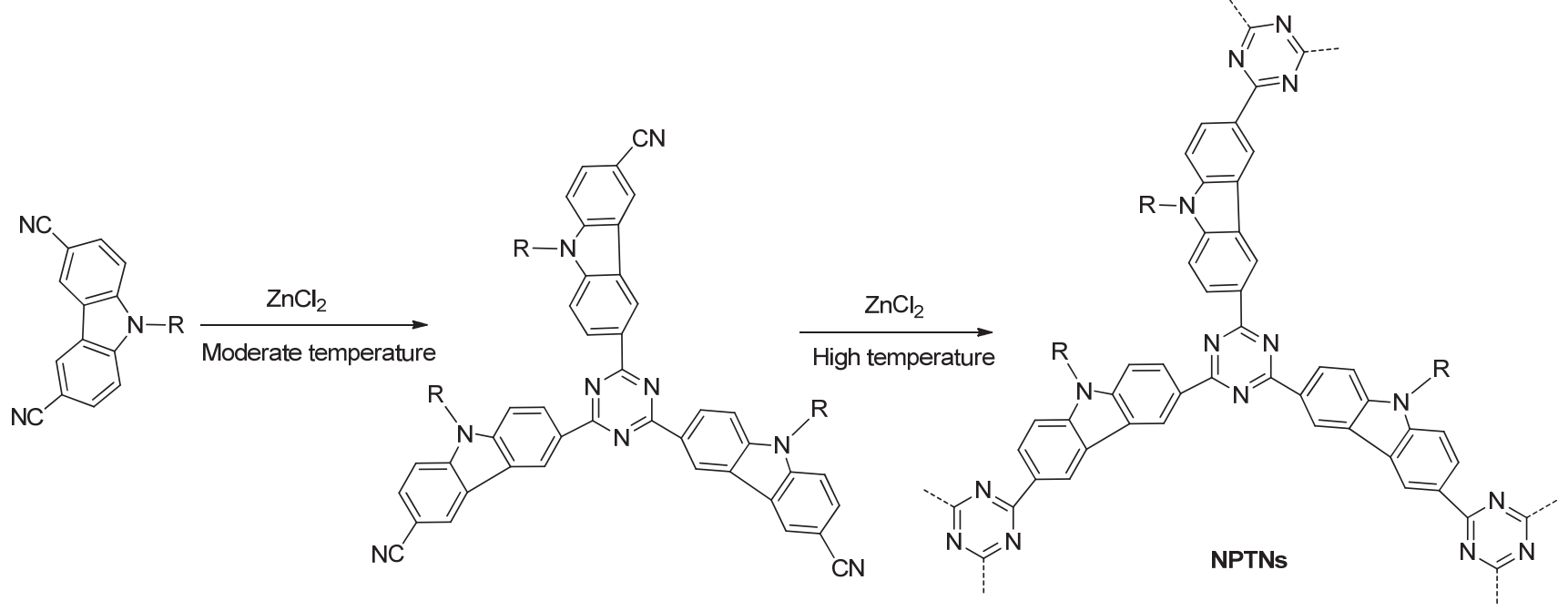

图式 11 聚咔唑 NPTNs 的合成

Scheme 11 Preparation of polymers NPTNs
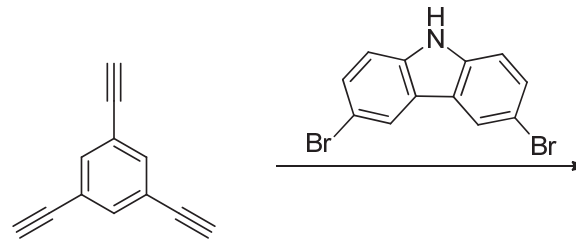

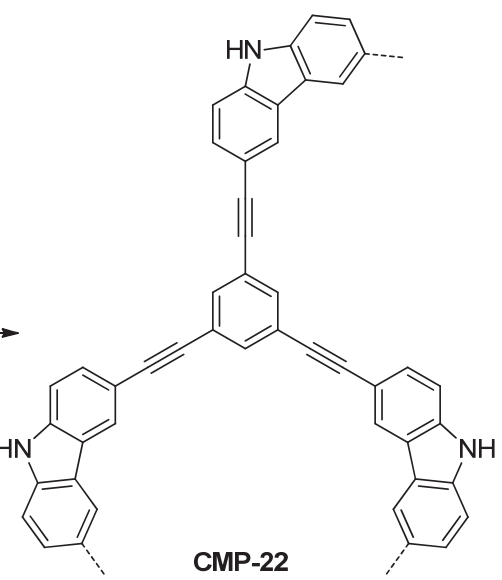

\subsubsection{Yamamoto 偶联反应}

2012 年, Jiang 课题组 ${ }^{[43]}$ 利用三聚咔唑衍生物通过 Yamamoto 偶联反应制备了一种含咔唑的新型共轭微孔 聚合物 TCB-CMP(图式 12), 并将其应用于芳香分子体 系的检测. 该共轭微孔聚合物呈现蓝色荧光, 并具有高 比表面积. 在苂光传感方面, 与其它线性结构的聚合物 相比, TCB-CMP 对芳烃蒸汽的快速检测具有较高的灵 敏度.

以上几种不同类型的多孔聚咔唑材料的制备方法 各有特点. 氧化偶联反应条件温和而且简单经济, 单体 结构一般需要含有两个以上的咔唑单元, 咔唑单元本身 即是反应基团, 无需引入其他官能团, 最终制备的材料 中没有其他基团的残留，结构更易表征. 碳一碳偶联反 应(Yamamoto 偶联反应、Sonogashira-Hagihara 偶联反应 等)应用范围较广, 一般都需要在单体中引入硼酸、卤 素、炔烃等基团, 比较适宜制备共轭有机多孔材料, 但 反应条件要求较高, 催化剂较贵, 不适宜于大量的制备. 超交联法制备多孔聚咔唑材料, 只需要在单体中引入交
联基团或外加交联剂就可以制备出种类多样的多孔材 料, 反应条件简单经济, 成本低, 比较适合多孔材料的 大量制备. 利用电化学聚合的制备方法，需要特定的合 成设备，比较适合制备多孔材料的薄膜.

\section{3 有机多孔聚咔唑的性能及应用}

有机多孔聚咔唑一般具有稳定的孔结构，比表面积 大多介于 $500 \sim 2400 \mathrm{~m}^{2} \cdot \mathrm{g}^{-1}$ 之间. 以孔径分布在 $0.5 \sim$ $7.0 \mathrm{~nm}$ 的微孔类型材料为主，通过孔道调节也可以制备 孔径分布在 $2.0 \sim 5.0 \mathrm{~nm}$ 的介孔型材料，而且它们的热 稳定性及化学稳定性很高, 因此在气体存储与分离、有 机蒸汽吸附、催化方面具有潜在的应用价值. 此外, 由 于部分共轭聚咔唑可以保持良好的光学电学性质, 具有 特异的光电性能, 这类多孔聚咔唑在化学生物传感和有 机电子学方面的应用也有新的突破.

\section{1 气体存储}

氢气是一种清洁能源，它具有较高的燃烧热(142 


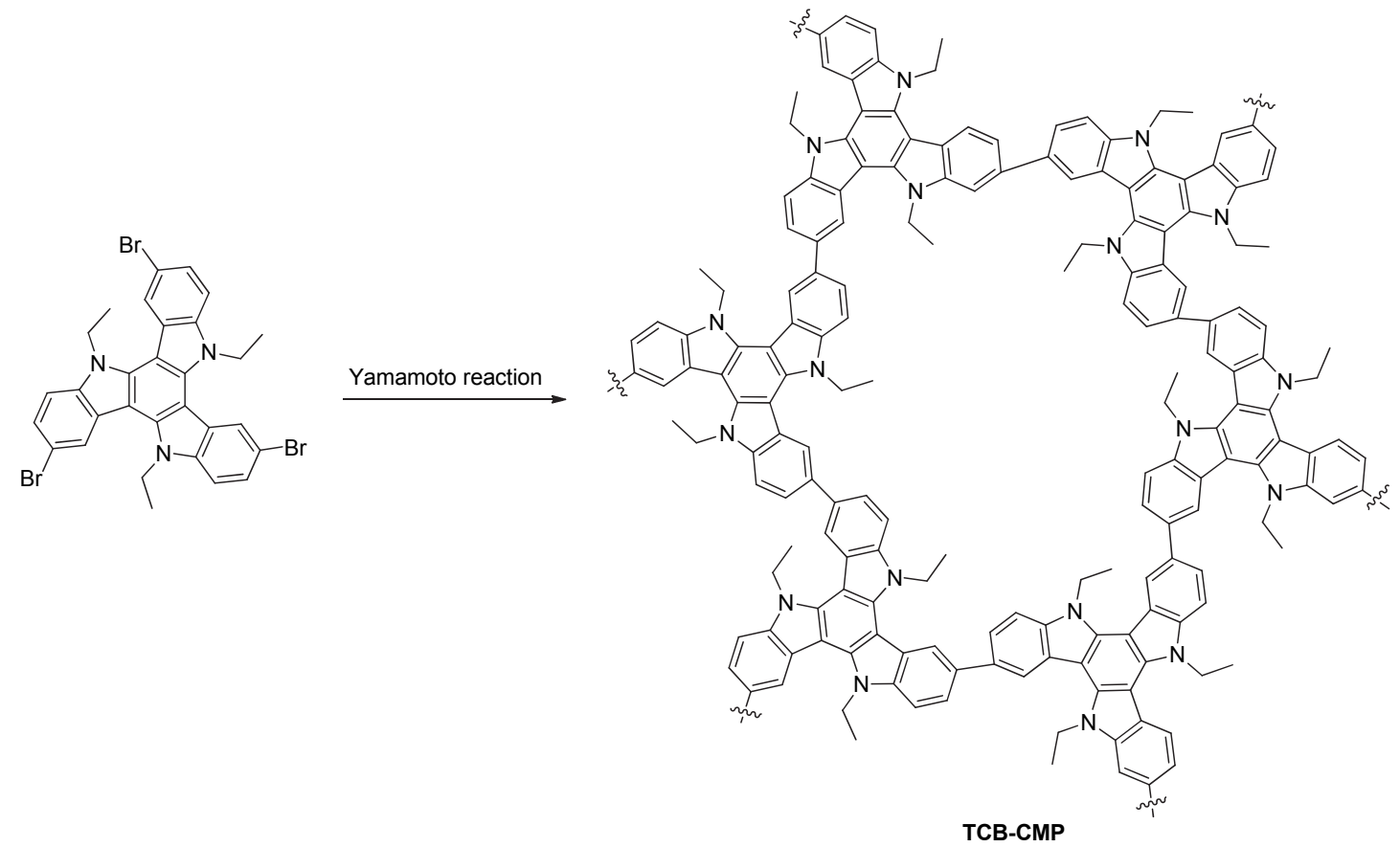

图式 12 通过 Yamamoto 偶联反应制备 TCB-CMP

Scheme 12 Preparation of TCB-CMP by Yamamoto coupling reaction

$\left.\mathrm{kJ} \cdot \mathrm{g}^{-1}\right)$, 是汽油 $\left(47.2 \mathrm{~kJ} \cdot \mathrm{g}^{-1}\right)$ 的 3 倍. 然而, 开发具有高容 量的储氢材料仍是一个重大的挑战. 有机多孔聚合物具 有较高的比表面积, 较窄的孔径分布以及较低的密度, 因此, 在储氢方面有应用潜力. 全球二氧化碳的排放量 在过去几十年里急剧上升, 被广泛认为是全球气候变暖 的主要原因之一. 通过碳捕获和储存 ${ }^{[23]}$ 的方法、进一步 利用二氧化碳成为降低大气二氧化碳含量的重要举措 之一. CCS 方法目前已被认为是最重要的途径之一, 而 对合成气和烟道气中的二氧化碳进行燃烧前捕集以及 燃烧后捕集是 CCS 技术的关键组成部分. 目前, 有机多 孔聚合物作为潜在的天然气储存或分离材料备受研究 工作者的关注. 聚咔唑作为一种很重要的含氮杂环的有 机多孔材料, 对热、酸和碱都比较稳定, 而且具有刚性 主链和共轭富电子体系, 既有利于形成永久性多孔材 料, 又可增强被吸附物与吸附剂之间的相互作用, 其含 氮结构可大大提高对酸性二氧化碳的吸附能力.

为了便于更好地了解对比有机多孔聚咔唑类材料 对气体的储存性能, 我们简单地总结了不同类型的多孔 材料比表面积及其对氢气和二氧化碳的吸附值(表 2). 部分已经报道的多孔聚咔唑显示了较高的储氢能力: 在 $1.0 \mathrm{bar}$ 和 $77 \mathrm{~K}$ 的条件下, CPOP-1 储氢可达 $2.80 \mathrm{wt} \%{ }^{[26]}$. CPOP-9 $\left(2440 \mathrm{~m}^{2} \cdot \mathrm{g}^{-1}\right) 、$ CPOP-18 $\left(1040 \mathrm{~m}^{2} \cdot \mathrm{g}^{-1}\right)$ 、 CPOP-19 $\left(1130 \mathrm{~m}^{2} \cdot \mathrm{g}^{-1}\right)$ 的储氢量分别为 $2.44 、 2.29$ 和 $2.39 \mathrm{wt} \%$. 此外, Cz-POF-1 $\left(2065 \mathrm{~m}^{2} \cdot \mathrm{g}^{-1}\right)$ 和 Cz-POF-3 $\left(1927 \mathrm{~m}^{2} \cdot \mathrm{g}^{-1}\right)$ 对氢气的吸附量也分别高达 2.24 和 2.07 $\mathrm{wt} \%$. 通过对比发现: 这些材料的氢气吸附能力要高于 大部分有机多孔材料, 而且可以与多孔碳材料和金属有 机骨架化合物相媲美. 在 $18.0 \mathrm{bar}$ 和 $77 \mathrm{~K}$ 的条件下, 咔
唑聚合物 CPOP-9 的氢气吸附性能可以和超高比表面积 的 PAF-1 $\left(5600 \mathrm{~m}^{2} \cdot \mathrm{g}^{-1}\right)$ 相比较, 吸附量分别为 5.22 和 $5.80 \mathrm{wt} \%$. 美国能源署设定的 2015 年氢燃料电池存储 目标是在工作温度为 $233 \sim 333 \mathrm{~K}$ 下最大输送压力为 $100 \mathrm{bar}$ 时达到重量容量 $5.5 \mathrm{wt} \%^{[44]}$. 目前, 它仍然是一 个巨大的挑战, 需要在实验中进一步探索出合适的储氢 材料.

在 $1.0 \mathrm{bar}$ 和 $273 \mathrm{~K}$ 的条件下, 有机多孔聚咔唑 CPOP-1 $\left(2220 \mathrm{~m}^{2} \cdot \mathrm{g}^{-1}\right)$ 对二氧化碳的吸附量高达 21.2 $\mathrm{wt} \%$, CPOP-9 对二氧化碳的吸附量为 $18.2 \mathrm{wt} \%$. 另外, Cz-POF-1 和 Cz-POF-3 对二氧化碳的吸附量也比较高, 分别为 20.2 和 $21 \mathrm{wt} \%$. 即使相比其他种类的具有高比 表面积的多孔材料, 这些聚咔唑多孔材料的二氧化碳吸 附性能也是比较突出的. 在 18.0 bar、 $298 \mathrm{~K}$ 的条件下, CPOP-9 对二氧化碳的吸附性能可以和超高比表面积的 PPN-4 ${ }^{[47]}\left(6461 \mathrm{~m}^{2} \cdot \mathrm{g}^{-1}\right)$ 相对比, 它们的吸附量分别为 70.0 和 $75.0 \mathrm{wt} \%$. 综上所述, 我们可以发现与其他类型 的多孔材料相比, 共轭聚咔唑多孔材料在气体吸附方面 具有优越的性能. 在低压或高压的条件下，这类聚咔唑 多孔材料都能表现出良好的吸附性能.

\section{2 蒸气吸附}

除了对气体的吸附外，有机多孔材料对一些小分子 有机蒸汽的吸附效果也很好, 这使得这种材料在环境方 面的应用有巨大潜力. 在常温和饱和蒸汽压下, CPOP-9 对甲苯吸附量高达 $1470 \mathrm{mg} \cdot \mathrm{g}^{-1}$, 高于具有超高比表面 积的 PAF-1 (1357 mg・g $\left.{ }^{-1}\right)$. 另外, CPOP-11 在选择性吸 附方面性能卓越，水和甲醇混合溶剂的选择性吸附质量 
表 2 低、高压力下的不同类型的多孔材料对气体的吸附性能

Table 2 Gas uptake capacities of various porous materials at different pressure

\begin{tabular}{|c|c|c|c|c|}
\hline Polymer & $S_{\mathrm{BET}} /\left(\mathrm{m}^{2} \cdot \mathrm{g}^{-1}\right)$ & $\mathrm{H}_{2}$ uptake $(1.0$ bar, $77 \mathrm{~K}) / \mathrm{wt} \%$ & $\mathrm{CO}_{2}$ uptake $(1.0 \mathrm{bar}, 273 \mathrm{~K}) / \mathrm{wt} \%$ & Ref. \\
\hline CPOP-1 & 2220 & 2.80 & 21.2 & [26] \\
\hline СРОР-7 & 1430 & 1.51 & 13.2 & [27] \\
\hline CPOP-8 & 1610 & 1.92 & 16.5 & [28] \\
\hline CPOP-9 & 2440 & $2.44 / 5.22^{a}$ & $18.2 / 70.0^{b}$ & [28] \\
\hline СРОР-13 & 890 & 1.25 & 16.8 & [37] \\
\hline CPOP-15 & 1190 & 1.29 & 14.2 & [37] \\
\hline CРОР-18 & 1040 & 2.29 & 15.1 & [38] \\
\hline СРОР-19 & 1130 & 2.39 & 16.7 & [38] \\
\hline $\mathbf{P}-2$ & 1222 & 1.66 & $14.5^{c}$ & [30] \\
\hline MFCMP-1 & 843 & - & 16.2 & [31] \\
\hline Cz-POF-1 & 2065 & $2.24 / 1.49^{d}$ & $20.2 / 12.9^{e}$ & [32] \\
\hline Cz-POF-3 & 1927 & $2.07 / 1.54^{d}$ & $21.0 / 13.4^{e}$ & [32] \\
\hline NPTF-1 & 1187 & - & 13.2 & [41] \\
\hline NPTN-1 & 1187 & - & $13.3 / 8.1^{d}$ & [42] \\
\hline TFMSA & 2250 & 1.7 & - & [36] \\
\hline TSP-2 & 913 & - & 18 & [39] \\
\hline BILP-1 & 1172 & 1.90 & 18.8 & [45] \\
\hline Trip(i-Pr)-PIM & 1601 & 1.83 & - & [46] \\
\hline PPN-3 & 2840 & 1.58 & - & [20] \\
\hline PPN-4 & 6461 & - & $75.0^{b}$ & [47] \\
\hline PPN-6-SO ${ }_{3} \mathrm{Li}$ & 1186 & - & 18.5 & [48] \\
\hline PAF-1 & 5600 & $1.50 / 5.80^{a}$ & 8.8 & [18] \\
\hline PAF-3 & 2932 & 2.07 & 15.3 & [49] \\
\hline MOP-C & 1237 & - & 17.0 & [50] \\
\hline COF-102 & 3530 & 1.2 & 5.3 & [51] \\
\hline Carbon AX-21 & 2421 & 2.40 & - & [52] \\
\hline Zeolite-like Carbon & 3200 & 2.60 & - & [53] \\
\hline MIL-101 & 5500 & 2.50 & - & [54] \\
\hline ZIF-69 & 1220 & - & 13.4 & [55] \\
\hline
\end{tabular}

${ }^{a}$ Data were obtained at 18.0 bar and $77 \mathrm{~K},{ }^{b}$ Data were obtained at 18 bar and $298 \mathrm{~K},{ }^{c}$ Data were obtained at 1.1 bar and $273 \mathrm{~K},{ }^{d}$ Data were obtained at 1.0 bar and $87 \mathrm{~K},{ }^{e}$ Data were obtained at 1.0 bar and $290 \mathrm{~K}$.

比高达 $1: 36\left(298 \mathrm{~K}, P / P_{0}=0.85\right)$, 可以用于去除水中 少量的甲醇. 此外, 我们还探索了一些多孔聚咔唑材料 对室内甲醛的吸附性能, 其中 CPOP-15 和 CPOP-19 对 甲醛吸附量(在室温和 $1 \mathrm{~atm}$ 的条件下)分别为 10.7 和 $11.2 \mathrm{mg} \bullet \mathrm{g}^{-1}$, 并且表现出良好的热稳定性和可重复性. 这使得该材料在消除环境中的有害的小分子化合物方 面具有较好的应用前景.

\section{3 异相催化}

对于化工和制药行业寻求绿色、安全、环保的技术 是当前科学研究的重点之一. 在过去的几十年里, 科研 工作者的目标是使用可回收的催化剂催化有机合成反 应, 以减少废弃物的产生和优化催化剂的效率. 有机多 孔材料作为一类具有纳米孔隙的高度交联无定形态的 聚合物，近年来在异相催化 ${ }^{[5]}$ 方面的应用备受关注.

有机多孔材料 MFCMP-1 ${ }^{[31]}$ 的内表面具有高浓度 的 Lewis 碱氮位点, 被用作 Knoevenagel 反应的催化剂, 对丙二腈与芳族化合物、杂环、环酮类具有高效的催化 作用(Eq. 2). 此外, 这种方法合成容易, 成本低, 再加上 多官能性, 使 MFCMP-1 在实际应用中具有很强的应用
前景.

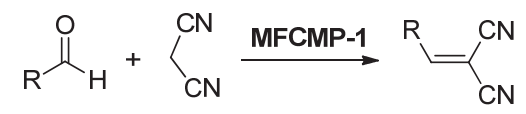

金属配位的卟啉在催化一些重要生化反应中起到 关键作用，而具有较高比表面积及较大孔容的有机多孔 聚合物为催化反应提供了良好的媒介. 我们课题组 ${ }^{[29]}$ 通过基于咔唑的氧化偶合聚合，合成具有特殊功能咔唑 卟啉多孔共轭聚合物 CPOP-11 和 CPOP-12. 其中含铁 卟啉多孔聚合物 CPOP-12 的 BET 比表面积为 1180 $\mathrm{m}^{2} \cdot \mathrm{g}^{-1}$, 可以应用于非均相催化, 能够高效催化硫代糖 苷的氧化来制备糖基亚砜(Eq. 3). 通过循环实验发现, 经过三次循环，催化剂的催化效果基本没有减弱，产率 都在 $80 \%$ 以上.

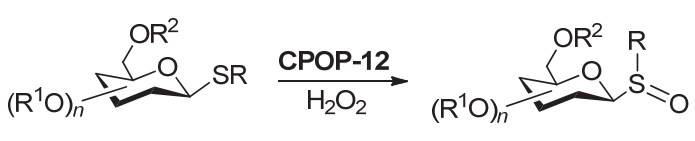

ca. $80 \%$ yield 


\section{4 传感}

有机多孔材料由于在修饰改性、光电性能的调节、 气体(或被测分子)选择性等方面均表现出明显的优势, 因此相应敏感器件的开发倍受关注. TCB-CMP ${ }^{[43]}$ 聚合 物对富电子芳烃蒸汽有突出的苂光增强效应, 而在缺电 子芳烃蒸汽环境下则产生强烈的苂光淬灭, 并且它可以 在灵敏度和响应性有所损失后还能够重复使用. 这些特 性归因于材料的共轭微孔性网络结构, 高比表面积的微 孔骨架结构不仅能够吸附芳烃分子使其进入聚合物的 空隙内, 而且还能促进激发态在框架内的迁移. 这些结 构特性的协同作用, 增强了 TCB-CMP 在检测过程中的 苂光激发与淬灭作用. 另外, 电化学聚合的方法能够可 控制备共轭多孔聚咔唑 TPBCz-CMP ${ }^{[34]}$ 的薄膜, 其厚度 可以控制, 并且可以用作衬底或独立的薄膜使用. 该薄 膜独特之处是具有多孔性, 延伸的 $p-\pi$ 共轭体系允许激 子离域骨架产生高速率的电子转移. 因此, 被开发用于 化疗和生物传感. 这种传感器具有良好的选择性, 快速 响应, 高灵敏度, 通过荧光开/关传感鉴别富电子和缺电 子芳烃以及通过氧化还原诱导荧光猝灭选择性检测氧 化离子.

\section{5 有机电子学}

随着共轭有机聚合物在电子学中研究的深入. 近年 来咔唑类有机多孔材料在电子学方面的应用 ${ }^{[33]}$, 逐渐 被人们所关注. 咔唑是一种高度电活化基团, 具有相对 低的氧化电位, 而且咔唑基团的独特性质能够精确控制 电化学聚合薄膜的结构. TPTCz 分子通过电化学氧化偶 联反应得到聚合物薄膜沉积到电极的表面. 电化学聚合 具有高的反应性以及咔唑完美的偶联反应使得到的薄 膜具有高度交联和确定的结构. 它拥有储存和释放抗衡 离子的能力, 并进一步影响了电性能. 掺杂这种薄膜的 电极层, 在聚合物太阳能电池中的功率转化效率为 $7.56 \%$, 在聚合物发光二极管中的发光效率为 20.7 $\mathrm{cd} \cdot \mathrm{A}^{-1}$, 这说明多孔共轭聚咔唑薄膜作为电极层在有机 电子领域中有巨大的潜力.

\section{4 结论与展望}

综上所述, 我们可以发现, 在短短的几年内, 含咔 唑的有机多孔材料发展迅速, 已经形成了一个基于咔唑 的有机多孔材料体系, 从最初的合成方法探究, 简单的 吸附以及分离应用, 发展到今天的共轭体系检测系统、 光学半导体、多种不同的气体(常见气体、稀有气体或 毒性气体)和蒸汽(有机有毒蒸汽)的吸附性能等方面的 应用探究. 这也反映了国内外有机多孔材料发展迅速的 态势. 当然, 有机多孔聚咔唑的相关研究工作在制备方 法学的发展、多孔性的有效调控、器件薄膜的制备以及 性能应用的拓展等方面还有很多值得探索和深入研究 的方向. 相信在不久的将来, 更多具有优异性能的材料 将被开发和应用. 基于咔唑的有机多孔材料的研究也会
对促进化学、材料科学以及更多其它领域的发展起到更 深远的影响.

\section{作者简介}

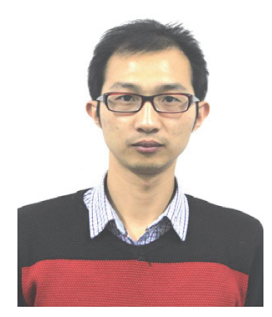

操强, 1988 年 12 月出生, 湖北黄冈人. 硕士就读于武汉工 程大学化工与制药学院, 主要从事过渡金属催化的 $\mathrm{C}-\mathrm{S}$ 键偶 联反应研究. 自 2014 年 6 月, 加入国家纳米科学中心韩宝航 研究员课题组, 从事有机聚合物的制备及相关性能的研究.

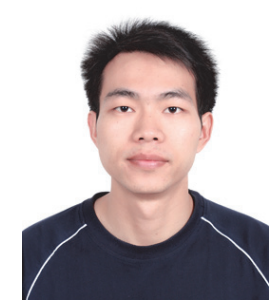

陈琦, 博士, 副研究员. 2007 年在中科院生态环境研究中 心获得理学博士学位. 先后在美国托莱多大学 (2007.5 2008.5)和国家纳米科学中心(2008.6 2010.3)从事博士后研究 工作. 2010.4 2012.3 任国家纳米科学中心助理研究员, 2012 年 4 月至今任国家纳米科学中心副研究员、中科院研究生院 硕士生导师, 中科院青年创新促进会会员. 研究方向为有机/ 高分子材料化学与物理, 目前主要从事有机多孔聚合物、糖功 能化有机分子的制备及相关性能和应用研究.

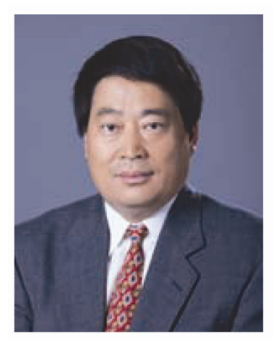

韩宝航, 博士, 研究员, 博士生导师. 南开大学学士学位 (1989)、硕士学位(1992)、博士学位(1999). 从 1992 年起分别 任南开大学化学系助教、讲师、副教授. 从 2000 年起分别在 德国马普胶体与界面研究所、加拿大渥太华大学和多伦多大 学从事博士后研究. 2005 年加入国家纳米科学中心, 从事超分 子纳米材料与器件方面的研究. 目前研究方向是纳米功能多 孔材料的制备与应用, 主要研究体系包括: (1)基于共轭单元的 有机(杂化)多孔功能材料; (2)基于石墨烯(氧化物)的多孔材料; (3)基于生物质的纳米多孔(碳)材料.

\section{References}

[1] Schüth, F.; Sing, K. S. W.; Weitkamp, J. Handbook of Porous Solids, Wiley-VCH, Weinheim, 2002.

[2] Xiong, J.-F.; Xiao, P.; Wu, Q.; Wang, X.-Z.; Hu, Z. Acta Chim. 
Sinica 2014, 72, 433. (熊静芳, 肖佩, 吴强, 王喜章, 胡征, 化学 学报, 2014, 72, 433.)

[3] Sui, D.; Huang, Y.; Huang, L.; Zhang, Y.; Chen, Y.-S. Acta Chim. Sinica 2014, 72, 382. (随东, 黄毅, 黄璐, 张昳, 陈永胜, 化学学 报, 2014, 72, 382.)

[4] Xiang, Z.-H.; Cao, D.-P. J. Mater. Chem. A 2013, 1, 2691.

[5] Zhang, Y. G.; Riduan, S. N. Chem. Soc. Rev. 2012, 41, 2083.

[6] Ding, S.-Y.; Wang, W. Chem. Soc. Rev. 2013, 42, 548.

[7] Cooper, A. I. CrystEngComm 2013, 15, 1483.

[8] Feng, X.; Ding, X.-S.; Jiang, D.-L. Chem. Soc. Rev. 2012, 41, 6010.

[9] Cote, A. P.; Benin, A. I.; Ockwig, N. W.; Keeffe, O. M.; Matzger, A. J.; Yaghi, O. M. Science 2005, 310, 1166.

[10] Zhang, T.-T.; Wang, H.-T.; Ma, H.-P.; Sun, F.-X.; Cui, X.-Q.; Zhu, G.-S. Acta Chim. Sinica 2013, 71, 1598. (张婷婷, 王海涛, 马和平, 孙福兴, 崔小强, 朱广山, 化学学报, 2013, 71, 1598.)

[11] McKeown, N. B.; Budd, P. M. Macromolecules 2010, 43, 5163.

[12] McKeown, N. B.; Budd, P. M. Chem. Soc. Rev. 2006, 35, 675.

[13] Budd, P. M.; Ghanem, B. S.; Makhseed, S.; McKeown, N. B.; Msayib, K. J.; Tattershall, C. E. Chem. Commun. 2004, 230.

[14] Xu, Y.-H.; Jin, S.-B.; Xu, H.; Nagai, A.; Jiang, D.-L. Chem. Soc. Rev. 2013, 428012.

[15] Cooper, A. I. Adv. Mater. 2009, 21, 1291.

[16] Tsyurupa, M. P.; Davankov, V. A. React. Funct. Polym. 2006, 66, 768.

[17] Ben, T.; Qiu, S.-L. CrystEngComm 2013, 15, 17.

[18] Ben, T.; Ren, H.; Ma, S.-Q.; Cao, D.-P.; Lan, J.-H.; Jing, X.-F.; Wang, W.-C.; Xu, J.; Deng, F.; Simmons, J. M.; Qiu, S.-L.; Zhu, G.-S. Angew. Chem., Int. Ed. 2009, 48, 9457.

[19] Wang, W.; Yan, Z.-J.; Yuan, Y.; Sun, F.-X.; Zhao, M.; Ren, H.; Zhu, G.-S. Acta Chim. Sinica 2014, 72, 557. (王维, 间卓君, 元野, 孙福兴, 赵明, 任浩, 朱广山, 化学学报, 2014, 72, 557.)

[20] Lu, W. G.; Yuan, D. Q.; Zhao, D.; Schilling, C. I.; Plietzsch, O.; Muller, T.; Bräse, S.; Guenther, J.; Blümel, J.; Krishna, R.; Li, Z.; Zhou, H. C. Chem. Mater. 2010, 22, 5964.

[21] Liu, D.-P.; Chen, Q.; Zhao, Y.-C.; Han, B.-H. Chin. Sci. Bull. 2013, 58, 2352. (刘德鹏, 陈琦, 赵彦超, 韩宝航, 科学通报, 2013, 58, 2352.)

[22] Zhao, Y.-C.; Chen, Q.; Han, B.-H. Sci. China Phys. Mech. Astron. 2011, 41, 1029. (赵彦超, 陈琦, 韩宝航, 中国科学: 物理学 力学 天文学, 2011, 41, 1029.)

[23] Haszeldine, R. S. Science 2009, 325, 1647.

[24] Dawson, R.; Laybourn, A.; Clowes, R.; Khimyak, Y. Z.; Adams, D. J.; Cooper, A. I. Macromolecules 2009, 42, 8809.

[25] Weber, J.; Thomas, A. J. Am. Chem. Soc. 2008, 130, 6334.

[26] Chen, Q.; Luo, M.; Hammershøj, P.; Zhou, D.; Han, Y.; Laursen, B. W.; Yan, C.-G.; Han, B.-H. J. Am. Chem. Soc. 2012, 134, 6084.

[27] Chen, Q.; Liu, D.-P.; Luo, M.; Feng, L.-J.; Zhao, Y.-C.; Han, B.-H. Small 2014, 10, 308.

[28] Chen, Q.; Liu, D.-P.; Zhu, J.-H.; Han, B.-H. Macromolecules 2014, $47,5926$.

[29] Feng, L.-J.; Chen, Q.; Zhu, J.-H.; Liu, D.-P.; Zhao, Y.-C.; Han, B.-H. Polym. Chem. 2014, 5, 3081.

[30] Qiao, S.-L.; Du, Z.-K.; Yang, R.-Q. J. Mater. Chem. A 2014, 2,
1877.

[31] Zhang, Y.-W.; Sigen, A.; Zou, Y.-C.; Luo, X.-L.; Li, Z.-P.; Xia, H.; Liu, X.-M.; Mu, Y. J. Mater. Chem. A 2014, 2, 13422.

[32] Zhang, X.; Lu, J.-Z.; Zhang, J. Chem. Mater. 2014, 26, 4023.

[33] Gu, C.; Chen, Y.-C.; Zhang, Z.-B.; Xue, S.-F.; Sun, S.-H.; Zhang, K.; Zhong, C.-M.; Zhang, H.-H.; Pan, Y.-Y.; Lv, Y.; Yang, Y.-Q.; Li, F.-H.; Zhang, S.-B.; Huang, F.; Ma, Y.-G. Adv. Mater. 2013, 25, 3443.

[34] Gu, C.; Huang, N.; Gao, J.; Xu, F.; Xu, Y.-H.; Jiang, D.-L. Angew. Chem., Int. Ed. 2014, 53, 4850.

[35] Katsoulidis, A. P.; Dyar, S. M.; Carmieli, R.; Malliakas, C. D.; Wasielewski, M. R.; Kanatzidis, M. G. J. Mater. Chem. A 2013, 1, 10465.

[36] Preis, E.; Widling, C.; Brunklaus, G.; Schmidt, J.; Thomas, A.; Scherf, U. ACS Macro. Lett. 2013, 2, 380 .

[37] Zhu, J.-H.; Chen, Q.; Sui, Z.-Y.; Pan, L.; Yu, J.-G.; Han, B.-H. J. Mater. Chem. A 2014, 2, 16181.

[38] Pan, L.; Chen, Q.; Zhu, J.-H.; Yu, J.-G.; He, Y.-J.; Han, B.-H. Polym. Chem. 2015, 6, 2478.

[39] Zhu, X.; Mahurin, S. M.; An, S. H.; DoThanh, C.; Tian, C. C.; Li, T. K.; Gill, L. W.; Hagaman, E. W.; Bian, Z. J.; Zhou, J. H.; Hu, J.; Liu, H. L.; Dai, S. Chem. Commun. 2014, 50, 7933.

[40] Saleh, M.; Lee, H. M.; Kemp, K. C.; Kim, S. K. ACS Appl. Mater. Interfaces 2014, 6, 7325.

[41] Liu, Y.; Wu, S. F.; Wang, G.; Yu, G. P.; Guan, J. G.; Pan, C. Y.; Wang, Z. G. J. Mater. Chem. A 2014, 2, 7795.

[42] Wu, S. F.; Liu, Y.; Yu, G. P.; Guan, J. G.; Pan, C. Y.; Du, Y.; Xiong, X.; Wang, Z. G. Macromolecules 2014, 47, 2875.

[43] Liu, X. M.; Xu, Y. H.; Jiang, D. L. J. Am. Chem. Soc. 2012, 134, 8738.

[44] Schlapbach, L.; Zuttel, A. Nature 2001, 414, 353.

[45] Rabbani, M. G.; El-Kaderi, H. M. Chem. Mater. 2011, 23, 1650.

[46] Ghanem, B. S.; Hashem, M.; Harris, K. D. M.; Msayib, K. J.; Xu, M.; Budd, P. M.; Chaukura, N.; Book, D.; Tedds, S.; Walton, A.; McKeown, N. B. Macromolecules 2010, 43, 5287.

[47] Yuan, D. Q.; Lu, W. G.; Zhao, D.; Zhou, H. C. Adv. Mater. 2011, 23, 3723.

[48] Lu, W.; Yuan, D.; Sculley, J.; Zhao, D.; Krishna, R.; Zhou, H.-C. J. Am. Chem. Soc. 2011, 133, 18126.

[49] Ben, T.; Pei, C.; Zhang, D.; Xu, J.; Deng, F.; Jing, X.; Qiu, S. L. Energy Environ. Sci. 2011, 4, 3991.

[50] Dawson, R.; Stockel, E.; Holst, J. R.; Adams, D. J.; Cooper, A. I. Energy Environ. Sci. 2011, 4, 4239.

[51] Furukawa, H.; Yaghi, O. M. J. Am. Chem. Soc. 2009, 131, 8875.

[52] Texier-Mandoki, N.; Dentzer, J.; Piquero, T.; Saadallah, S.; David, P.; Vix-Guterl, C. Carbon 2004, 42, 2744.

[53] Yang, Z. X.; Xia, Y. D.; Mokaya, R. J. Am. Chem. Soc. 2007, 129, 1673 .

[54] Latroche, M.; Surble, S.; Serre, C.; Mellot-Draznieks, C.; Llewellyn, P. L.; Lee, J. H.; Chang, J. S.; Jhung, S. H.; Ferey, G. Angew. Chem., Int. Ed. 2006, 45, 8227.

[55] Banerjee, R.; Phan, A.; Wang, B.; Knobler, C.; Furukawa, H.; O’Keeffe, M.; Yaghi, O. M. Science 2008, 319, 939 